\title{
Fluiddynamik in der Cigarette und deren Einfluß auf den Zugwiderstand*
}

\author{
Horst Gaißer, \\ BAT-CF Hamburg, \\ Babrenfelder Chaussee, 139 \\ D-22761 Hamburg 50.
}

\section{SUMMARY}

The objective of the theoretical study is to present a model that simulates the instationary flow characteristics within cigarettes which are adjusted to a routine smoking machine. By assuming laminar fluid flow the well known KOZENY-CARMAN equation is used to evaluate the flow impedance of cigarettes.

The model parameters are determined by five variations of routine pressure-drop measurements yielding the entire set of flow impedances, which are necessary for describing a steady state Fluid-Network.

Using this relation further elements of the fluid dynamics, e.g. the Fluid-Inductor, a storage element of kinetic energy (fluid inertor), and the Fluid-Capacitor, a storage element of potential energy (fluid volume), are evaluated. In addition, the electrical and fluid dynamical analogies are demonstrated.

The model uses a set of distributed parameters including pneumatic impedances, capacitors and inductors, to represent filtered cigarettes and a second order linear partial differential equation to describe the fluid flow within the cigarettes. A simple presentation of the Fluid-Network with lumped parameters is analyzed and its significant time constants are determined. A characteristical value for the time constant of the cigarette fluid-inertor is given by $\mathrm{T}_{\mathrm{L}} \approx 0.02 \mathrm{~ms}$ and

\footnotetext{
* Vortrag anläßlich des 35. Tabak-Kolloquiums in Bremen, 6. Juni - 11. Juni 1993. Eingegangen: 28. Mai 1993 - angenommen: 12. Seprember 1993
}

depending on the fluid volume of cigarette their dominant time parameter is given by $\mathrm{T}_{c} \approx 2 \mathrm{~ms}$.

Finally the transient fluid flow within cigarettes in a conventional smoking machine is considered including a simulation of their fluid dynamics. A simple estimation gives the time response of the system (consisting of cigarettes and routine smoking machine) with $\mathrm{T}_{\mathrm{cR}} \approx$ $70 \mathrm{~ms}$ as a product of dead and sweep volume times the pressure drop of unencapsulated cigarettes. Variation of parameters caused by cigarette pressure drops and dead volumes of the smoking machine are simulated and discussed.

\section{ZUSAMMENFASSUNG}

Gegenstand der vorgestellten Untersuchung ist der instationäre Strömungszustand in Cigaretten in Verbindung mit der Routine-Abrauchmaschine.

Zur Bestimmung der Strömungsimpedanz einer Cigarette gilt die KOZENY-CARMAN-Gleichung für laminare Strömungen.

Die Parameterbestimmung geschieht durch fünf Varianten der herkömmlichen Zugwiderstandsmessung und liefert alle Strömungsimpedanzen zur Beschreibung eines stationären Fluidnetzwerkes.

Darauf aufbauend werden weitere Elemente der Fluiddynamik, die Fluid-Induktivität als kinetische Energie speicherndes Element (bewegte Fluidmasse) und die Fluid-Kapazität als potentielle Energie speicherndes Element (Fluidvolumenspeicher) entwickelt sowie all- 
gemeine elektrische und strömungsmechanische Analogien aufgezeigt.

Die Filter-Cigarette wird hier zunächst mit verteilten Parametern charakterisiert, d.h. mit Strömungsimpedanz, Fluid-Kapazität und -Induktivität modelliert und mit einer linearen, partiellen Differentialgleichung 2. Ordnung beschrieben. Eine einfache Darstellung durch ein Fluidnetzwerk mit konzentrierten Parametern wird analytisch untersucht und signifikante Zeit konstanten ermittelt. Als charakteristische Zeitkonstante der bewegten Fluidmasse erhält man $T_{L} \approx 0,02$ ms und infolge des Volumenspeichers in der Cigarette die dominierende Kenngröße $\mathrm{T}_{\mathrm{c}} \approx 2 \mathrm{~ms}$.

Abschließend wird das Strömungsverhalten der Cigarette in der Abrauchmaschine untersucht und die instationären fluidmechanischen Vorgänge simuliert. Eine einfache Abschätzung für die Zeitkonstante des Systems Cigarette-Rauchmaschine ergibt $T_{c R} \approx 70 \mathrm{~ms}$ als Produkt von Tot-/Hub-Volumen mal Zugwiderstand der offenen Cigarette. Parametereinwirkungen durch Variation des Zugwiderstands der Cigarette und des Totvolumens der Abrauchmaschine werden simuliert.

\section{RESUME}

Cette étude théorique présente un modèle qui simule les caractéristiques d'un courant non stationnaire à l'intérieur des cigarettes reliées à une machine à fumer classique. Pour évaluer l'impédence du courant gazeux dans une cigarette, on utilise l'équation de KOZENYCARMAN s'appliquant aux écoulements laminaires des fluides.

La détermination des paramètres s'effectue à l'aide de cinq variantes du procédé de mesure classique de la chute de pression et fournit toutes les impédances nécessaires pour d'écrire un réseau de fluide à l'état stationnaire.

Partant de là, on peut définir d'autres éléments de la dynamique des fluides, l'inductance par exemple, élément de stockage de l'énergie cinétique (masse du fluide en mouvement) et la capacité, élément de stockage de l'énergie potentielle (volume du fluide) et mettre en évidence des analogies d'ordre général avec l'électricité et la mécanique des fluides.

Le modèle fait appel à des paramètres répartis (impédance du courant gazeux, capacité et inductance) pour représenter les cigarettes à filtre et à une équation différentielle partielle linéaire du second ordre pour décrire l'écoulement du fluide à l'intérieur des cigarettes. On a analysé une représentation simple du réseau à l'aide de paramètres concentrés et déterminé ses constantes de temps significatives. Une valeur caractéristique de la constante de temps de la masse de fluide en mouvement est donnée par $T_{L}=0,02 \mathrm{~ms}$, et il résulte du volume de fluide dans la cigarette une caractéristique dominante correspondant à $\mathrm{T}_{\mathrm{c}}=2 \mathrm{~ms}$. Pour terminer, on a examiné le comprtement des cigarettes dans une machine à fumer classique et simulé un régime non stationnaire. Une estimation simple de la constante de temps du système cigarette/machine à fumer conduit à la valeur $\mathrm{T}_{\mathrm{CR}}=70 \mathrm{~ms}$ résultant du produit du volume mort total/de la course par la résistance au tirage de la cigarette non encapsulée. Les variations des paramètres provoquées par les chutes de pression dans la cigarette et le volume mort total de la machine à fumer ont été simulées et discutées.

\section{EINLEITUNG}

Die Bestimmung des Druckverlustes ("Zugwiderstand") zur Untersuchung von Tabak und Tabakerzeugnissen (Cigaretten) erfolgt bei stationärer Durchströmung mit einem "Normzug" $Q_{N Z}=17,5 \mathrm{ml} / \mathrm{s}$.

In (1) wird der stationäre Strömungszustand in FilterCigaretten mit Strang- und Filterventilation beschrieben.

Für laminare Strömungen ist nach HAGEN-POISEULLE der Druckverlust $P$ proportional dem Volumenstrom $Q$ und es gilt die KOZENY-CARMAN-Gleichung zur Bestimmung des Strömungswiderstandes.

Darauf aufbauend wurden in (1) zwei Modelle vorgestellt:

a) stationäres Modell mit verteilten Parametern (Modellierung mit verteilten Parametern, d.h. Strömungsimpedanz pro Längeneinheit und mathematische Beschreibung mittels Differentialgleichungen),

b) stationäres Modell mit diskreten Parametern (Modellierung mit diskreten Parametern, d.h. Kettenschaltung von T-Vierpol-Strömungsimpedanzen und mathematische Beschreibung mittels Differenzengleichungen).

Die Strömungsgrößen wie Druckverlust $P(1 \mathrm{~Pa} \approx 0,1$ mmWS), Volumenstrom $Q\left(1 \mathrm{~m}^{3} / \mathrm{s}=10^{6} \mathrm{ml} / \mathrm{s}\right)$ und Strömungsimpedanz $Z$ (1 $\left.\mathrm{Pa} \mathrm{s} / \mathrm{m}^{3}=10^{-6} \mathrm{~Pa} \mathrm{~s} / \mathrm{ml}\right)$ sind von der Ortskoordinate $x$ abhängig.

Als herausragende, meßtechnisch ermittelbare invariante Modellkenngröße erhält man den Strömungswellenwiderstand $Z_{W}$ und die Fortpflanzungskonstante $\gamma$. In der Routine-Abrauchmaschine ist die "warme" Cigarette mit Glutkegel dynamischen Strömungszuständen ausgesetzt (2). Dieser Prozeß hat als Eingangsgröße ein glockenförmiges Zugvolumen $(35 \mathrm{ml}$ bei einer Zugdauer von 2 s) und als Systemantwort ein Zugprofil, das zeitlich verzögert ist, sowie ein asymp- 
totisches Eigenverhalten nach der Zugdauer aufweist. Dieses Verhalten wird nach einer Modellierung für instationäre Strömung untersucht.

\section{STATIONÄRE STRÖMUNGSVERHÄLTNISSE}

Der Zugwiderstand (Druckverlust, draw resistance, pressure drop) ist die nach 3 bis 5 Sekunden Durch-

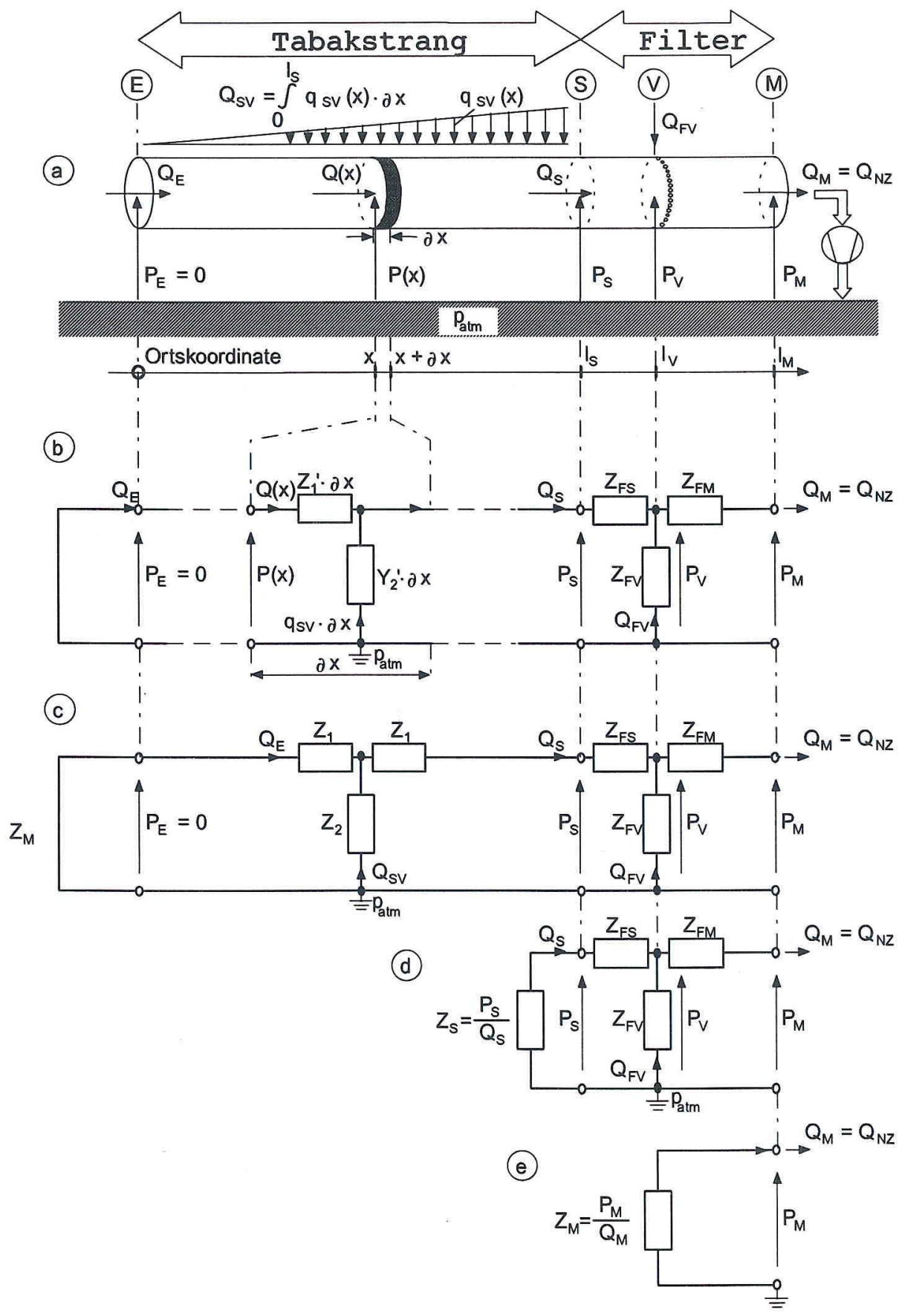

Abbildung 1.

Stationäres Modell einer "kalten" Filter-Cigarette mit Normzug $Q_{\mathrm{NZ}}=17,5 \mathrm{ml} / \mathrm{s}$ und Druckverlust

(Zugwiderstand, pressure drop) $\mathrm{P}_{\mathrm{M}}$;

a) Wirkschaltbild mit Volumenstrom $Q$ und Unterdruck $P$,

b) Fluidnetzwerk mit verteilten Strömungsimpedanzen $Z_{1}{ }^{\prime}, Z_{2}{ }^{\prime}=1 / Y_{2}{ }^{\prime}$ für den Tabakstrang,

c) Fluidnetzwerk mit einem diskreten T-Vierpol für den Tabakstrang,

d) Fluidnetzwerk mit Ersatz-Strömungsimpedanz $Z_{S}$ für den Tabakstrang,

e) Ersatz-Strömungsimpedanz $Z_{M}$ für die gesamte Filter-Cigarette. 
strömungsdauer (stationäre Durchströmung) vorhandene Differenz der statischen Drücke zwischen dem Eintrittsende und dem Austrittsende, wenn die Cigarette in waagerechter Lage von einem stationären Luftstrom von $17,5 \mathrm{ml} / \mathrm{s}$, bezogen auf das Austrittsende, durchströmt wird (3). Am Mundstück ( $M$ ) einer "kalten" Filter-Cigarette wird ein Normzug $Q_{N Z}=17,5$ $\mathrm{ml} / \mathrm{s}$ angelegt (Abbildung $1 \mathrm{a}$ ).

Am Eintrittsende $(E)$ ist der Unterdruck $P_{E}=0$ (Atmosphäre) und der Axialstrom beträgt $Q_{E}$. Über die
Tabakstranghülle fließt ein verteilter Querstrom (Strangventilation) $Q_{S V}$, der sich mit dem Axialstrom vermischt, so daß letzterer bis zur Trennstelle Tabakstrang-Filter $(S)$ auf $Q_{S}$, bei einem (Unter-) Druck $P_{S}$, angewachsen ist. Die Axialströmung im Filter ist bis zur Ventilationszone $(V)$ konstant und steigt dort sprunghaft, um den Ventilationsstrom $Q_{F V}$ an, bei einem (Unter-) Druck $P_{V}$. Die Axialströmung bleibt dann bis zum Austrittsende, dem Filtermundstück $(M)$ konstant, mit $Q_{M}=Q_{N Z}$ und dem maximalen (Unter-) Druck $P_{M}$, dem sogenannten "Zugwiderstand".

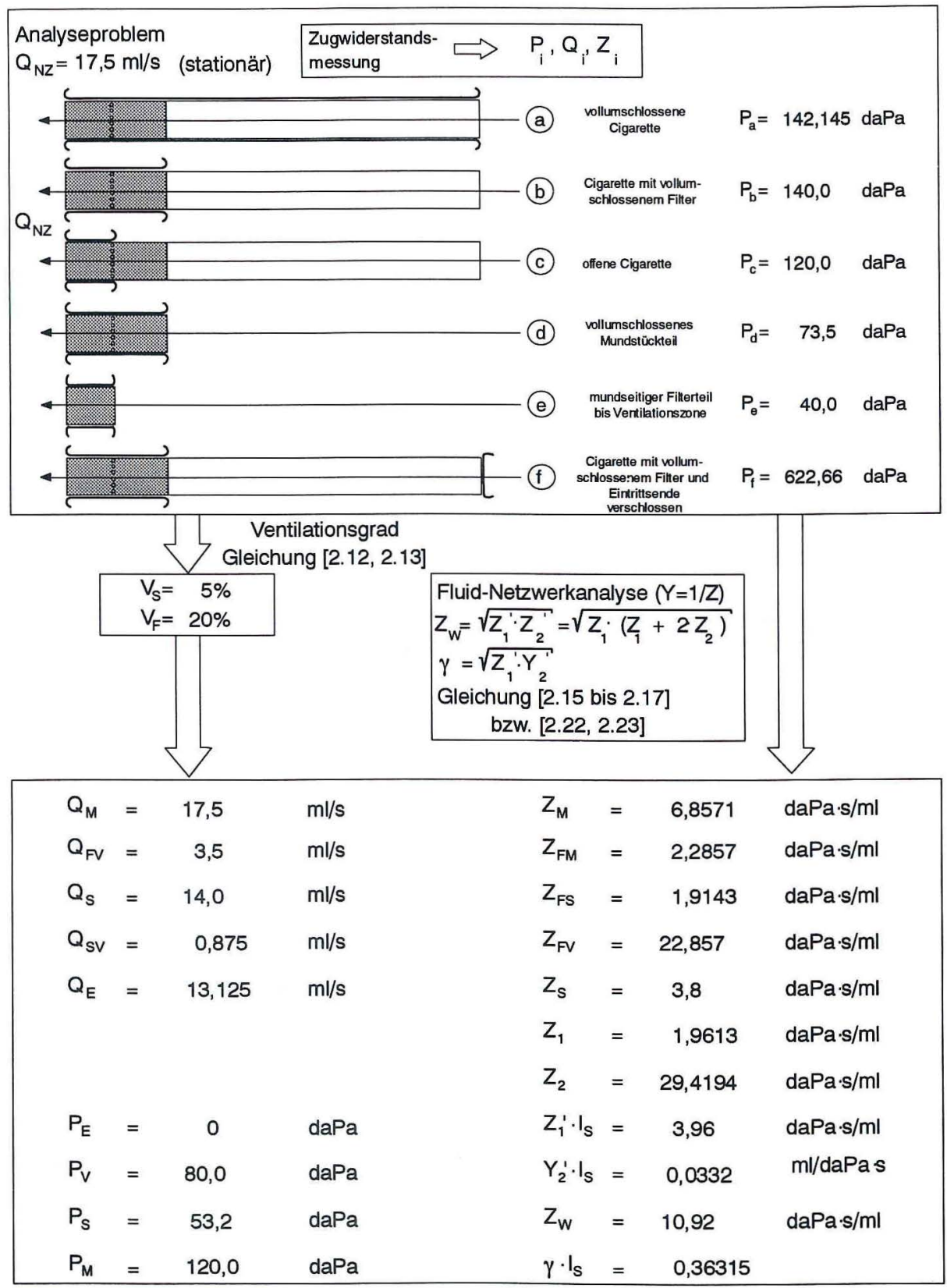

Abbildung 2.

Druckverlustmessung an einer "kalten" fiktiven Filter-Cigarette im King-Size-Format und daraus bestimmte FluidNetzwerkparameter $(1 \mathrm{daPa} \approx 1 \mathrm{mmWS})$. 
Die Filter-Cigarette läßt sich für diese stationären Strömungsverhältnisse durch Fluid-Netzwerke modellieren und beschreiben, sei es detailliert durch verteilte Strömungsimpedanzen für den Tabakstrang oder global durch die Ersatz-Strömungsimpedanz $Z_{M}$ (Abbildung $1 \mathrm{~b}$ bis $1 \mathrm{e}$ ).

Eine ventilierte Filter-Cigarette wird durch Variation der herkömmlichen Zugwiderstandsmessungen analysiert. Die gemessenen Druckverluste werden mit

$P_{a}: \quad$ vollumschlossene Cigarette,

$P_{b}$ : $\quad$ Cigarette mit vollumschlossenem Filter,

$P_{c}: \quad$ offene Cigarette $\left(P_{c}=P_{M}\right.$, in Abbildung 1),

$P_{d}: \quad$ vollumschlossenes Mundstückteil,

$P_{e}: \quad$ mundseitiger Filterteil bis Ventilationszone

bezeichnet und sind aus Abbildung 2 ersichtlich ( $P: 1$ $\mathrm{daPa} \approx 1 \mathrm{mmWS}$ ).

Für diese fünf Zugwiderstandsmessungen ergeben sich wiederum (mit den Fluid-Netzwerken nach Abbildung 1) folgende Abhängigkeiten:

$$
\begin{gathered}
P_{a}=Q_{N Z} \cdot\left(Z_{F M}+Z_{F S}+2 Z_{1}\right) \\
P_{b}=Q_{N Z} \cdot\left(Z_{F M}+Z_{F S}+Z_{S}\right) \\
P_{M}=P_{c}=Q_{N Z} \cdot Z_{M} \\
P_{d}=Q_{N Z} \cdot\left(Z_{F M}+Z_{F S}\right) \\
P_{e}=Q_{N Z} \cdot Z_{F M}
\end{gathered}
$$

Die Ersatz-Strömungsimpedanz ist für den Tabakstrang

$$
Z_{S}=Z_{1}+\frac{Z_{1} \cdot Z_{2}}{Z_{1}+Z_{2}}
$$

und für die gesamte Filter-Cigarette

$$
Z_{M}=Z_{F M}+\frac{Z_{F V} \cdot\left(Z_{F S}+Z_{S}\right)}{Z_{F V}+Z_{F S}+Z_{S}}
$$

Mit dem Filterventilationsgrad $V_{F}$

$$
Q_{F V}=V_{F} \cdot Q_{N Z}
$$

und dem Tabakstrangventilationsgrad $V_{S}$

$$
Q_{S V}=V_{S} \cdot Q_{N Z}
$$

erhält man durch elementares Ablesen der Strömungsbilanzen und entsprechendes Ausrechnen nach Gleichung [2.1 bis 2.9] alle gewünschten Parameter des Fluid-Netzwerkes. Die Charakterisierung einer Filterzigarette durch fünf Fluidkennzahlen wurde in einem Vortrag anläßlich des 36. Tabak-Kolloquiums in Lübeck, 5. Juni bis 10. Juni 1994, vorgestellt (Charakteristische Fluidkennzahlen im Parameterraum $R^{5}$ ). In Abbildung 2 sind diese für das schon in (1) benutzte Kanonische Zahlenbeispiel einer fiktiven "kaIten" ventilierten Filter-Cigarette im King-Size-Format zusammengestellt. Aus der Maschengleichung folgt z.B.

$$
\begin{aligned}
P_{M} & =P_{c}=P_{S}+Z_{F S} \cdot Q_{S}+Z_{F M} \cdot Q_{N Z} \\
& =P_{S}+P_{d} \cdot\left(1-V_{F}\right)+P_{e} \cdot V_{F} .
\end{aligned}
$$

Die Tabakstrang-Ersatzströmungsimpedanz lautet dann

$$
\begin{aligned}
Z_{S} & =\frac{P_{S}}{Q_{S}}=\frac{P_{c}-P_{d} \cdot\left(1-V_{F}\right)-P_{e} \cdot V_{F}}{\left(1-V_{F}\right) \cdot Q_{N Z}} \\
& =\frac{P_{b}-P_{d}}{Q_{N Z}}=\frac{P_{a}-P_{d}}{2 \cdot Q_{N Z}} \cdot\left(2-\frac{V_{s}}{1-V_{F}}\right),
\end{aligned}
$$

der Filterventilationsgrad

$$
V_{F}=\frac{P_{b}-P_{c}}{P_{b}-P_{e}}
$$

und der Strangventilationsgrad

$$
\begin{aligned}
V_{s} & =2 \cdot\left(1-V_{F}\right) \cdot \frac{\boldsymbol{P}_{a}-\boldsymbol{P}_{b}}{\boldsymbol{P}_{a}-\boldsymbol{P}_{d}} \\
& =2 \cdot \frac{\boldsymbol{P}_{c}-\boldsymbol{P}_{e}}{\boldsymbol{P}_{b}-\boldsymbol{P}_{e}} \cdot \frac{\boldsymbol{P}_{a}-\boldsymbol{P}_{b}}{\boldsymbol{P}_{a}-\boldsymbol{P}_{d}} .
\end{aligned}
$$

Weiterhin wurde in (1) die Ersatz-Strömungsimpedanz des Tabakstrangs $Z_{S r}=Z_{S}\left(l_{S r}, Z_{G}\right)$ allgemein in Abhängigkeit der reduzierten Tabakstranglänge $l_{S r}\left(0 \leq l_{S r}\right.$ $\leq l_{s}=63 \mathrm{~mm}$ ) und der Glutkegelimpedanz $Z_{G}$ für die brennende Cigarette ermittelt

$$
Z_{S r}=Z_{S}\left(l_{S r}, Z_{G}\right)
$$

$$
=Z_{W} \cdot \frac{\frac{Z_{G}}{Z_{W}} \cdot \operatorname{ch}\left(\gamma \cdot l_{S r}\right)+\operatorname{sh}\left(\gamma \cdot l_{S r}\right)}{\frac{Z_{G}}{Z_{W}} \cdot \operatorname{sh}\left(\gamma \cdot l_{S r}\right)+\operatorname{ch}\left(\gamma \cdot l_{S r}\right)} .
$$


Hierbei ist das Strömungsverhältnis im kalten Tabakstrang

$$
\begin{aligned}
\frac{Q_{S}}{Q_{E}} & =\frac{1-V_{F}}{1-\left(V_{F}+V_{S}\right)} \\
& =\frac{P_{a}-P_{d}}{2 \cdot P_{b}-P_{a}-P_{d}},
\end{aligned}
$$

die Fortpflanzungskonstante (Ausbreitungskonstante) $\gamma$

$$
\gamma \cdot l_{S}=\operatorname{arch}\left(\frac{Q_{S}}{Q_{E}}\right),
$$

der Strömungswellenwiderstand $Z_{W}$

$$
Z_{W}=\frac{P_{b}-P_{d}}{Q_{N Z}} \cdot \frac{\operatorname{ch}\left(\gamma \cdot l_{S}\right)}{\operatorname{sh}\left(\gamma \cdot l_{S}\right)},
$$

sh, ch, th bzw. arch sind die hyperbolischen Funktionen Sinushyperbolicus, Cosinushyperbolicus, Tangenshyperbolicus bzw. Areacosinushyperbolicus $(y=\operatorname{arch}$ (x) für $x=\operatorname{ch}(y))$.

Die Fortpflanzungskonstante $\gamma$ und der Strömungswellenwiderstand $Z_{W}$ des Tabakstranges kann auch über die Messung der "Leerlauf- und Kurzschlußimpedanz" (1) bestimmt werden. Um die Empfindlichkeit der herkömmlichen Zugwiderstands-Meßgeräte optimal auszunutzen, wird diese Messung, falls erforderlich, mit einem reduzierten Tabakstrang $\left(0<l_{s r} \leq l_{S}\right)$ durchgeführt (Index $r$ ).

Bei der reduzierten Filter-Cigarette mit vollumschlossenem Filter gilt für den gemessenen "KurzschlußDruckverlust" $P_{b r}$

$$
P_{b r}=Q_{N Z} \cdot\left(Z_{F M}+Z_{F S}+Z_{S K r}\right)
$$

mit der "Kurzschluß-Strömungsimpedanz" $Z_{S K r}$ des reduzierten Tabakstranges (Eintrittsende $\mathrm{E}$ offen, d.h. $Z_{G}=0$ in [2.14])

$$
Z_{S K r}=Z_{S}\left(l_{S r}, Z_{G}=0\right)=Z_{W} \cdot \operatorname{th}\left(\gamma \cdot l_{S r}\right) .
$$

Ist die reduzierte Filter-Cigarette mit vollumschlossenem Filter zusätzlich am Eintrittsende verschlossen, gilt für den gemessenen "Leerlauf-Druckverlust" $P_{f r}$

$$
P_{f r}=Q_{N Z} \cdot\left(Z_{F M}+Z_{F S}+Z_{S L r}\right),
$$

mit der "Leerlauf-Strömungsimpedanz" $Z_{S L r}$ des reduzierten Tabakstranges (Eintrittsende $\mathrm{E}$ geschlossen, d.h. $Z_{G} \rightarrow \infty$ in [2.14\})

$$
Z_{S L r}=Z_{S}\left(l_{S r}, Z_{G} \rightarrow \infty\right)=Z_{W} \cdot \frac{1}{\operatorname{th}\left(\gamma \cdot l_{S r}\right)} .
$$

Wird die Druckverlustmessung $P_{d}$ des vollumschlossenen Mundstückes nach Gleichung [2.4] berücksichtigt, erhält man den Wellenwiderstand $Z_{W}$

$$
\begin{aligned}
Z_{W} & =\sqrt{Z_{S L r} \cdot Z_{S K r}} \\
& =\frac{1}{Q_{N Z}} \cdot \sqrt{\left(P_{f r}-P_{d}\right) \cdot\left(P_{b r}-P_{d}\right)}
\end{aligned}
$$

und die Fortpflanzungskonstante

$$
\operatorname{th}\left(\gamma \cdot l_{S r}\right)=\sqrt{\frac{Z_{S K r}}{Z_{S L r}}}=\sqrt{\frac{P_{b r}-P_{d}}{P_{f r}-P_{d}}} .
$$

In Abbildung 2 ist der Tabakstrang ungekürzt $\left(l_{S r}=\right.$ $l_{s}$ ) dargestellt mit den Meßwerten $P_{b r}=P_{b}=140 \mathrm{daPa}$ und $P_{f r}=P_{f}=622,66 \mathrm{daPa}$.

Durch die Substitution von $Z_{S r} \rightarrow Z_{S}$ in Gleichung $[2.2],\{2.7\}$ sind alle Parameter, auch für die brennende Cigarette mit der Reststranglänge $l_{S r}$ und Glutkegelimpedanz $Z_{G}$, bestimmt.

\section{ELEMENTE DER FLUIDDYNAMIK}

Ein Strömungszustand ist eindeutig beschrieben, wenn die Strömungsgeschwindigkeit $\vec{v}$ und der Druck $p$ in Abhängigkeit vom Ort $\overrightarrow{\mathrm{r}}$ und der Zeit $t$ bekannt ist. Das Grundgesetz von NEWTON in der EULERschen Form lautet: "Summe der äußeren Kräfte gleich Masse mal Beschleunigung"

$$
\overrightarrow{\mathrm{k}}_{(\overrightarrow{\mathrm{r}}, t)}=m \cdot \frac{\mathrm{d} \overrightarrow{\mathrm{v}}(\overrightarrow{\mathrm{r}}, t)}{\mathrm{d} t} .
$$

Dies auf ein Fluidteilchen angesetzt führt auf die EULERsche Bewegungsgleichung ((1), Kap. 2.1) bzw. bei einem Stromfaden $\overrightarrow{\mathrm{v}}(\overrightarrow{\mathrm{r}}, t) \rightarrow v(s, t)$ auf die BERNOULLIsche Gleichung für instationäre Bewegung

$$
\begin{gathered}
\frac{v_{1}^{2}}{2 g}+\frac{p_{1}}{\gamma}+z_{1}= \\
\frac{v_{2}^{2}}{2 g}+\frac{p_{2}}{\gamma}+z_{2}+\frac{1}{g} \int_{s_{1}}^{s_{2}} \frac{\delta v}{\delta t} \mathrm{~d} s .
\end{gathered}
$$

Hierbei bedeuten $v$ die Geschwindigkeit, $g$ die Erdbeschleunigung, $p$ der Druck, $\gamma=\rho \cdot g$ das spezifi- 
sche Gewicht, und $z$ die über einem beliebig gewählten Bezugsniveau gerechnete Höhe.

Mit der Kontinuitätsgleichung für den Stromfaden

$$
Q=A_{1} \cdot v_{1}=A_{2} \cdot v_{2}=\text { const. }
$$

beherrscht [3.2] die instationären Strömungsvorgänge, sofern die zur Querschnittsfläche $A$ senkrechte Geschwindigkeit ïber $A$ als konstant angesehen werden kann. Es fehlt nun noch die Zustandsgleichung, eine Beziehung zwischen der Dichte $\rho$, der Temperatur $\vartheta$ und dem Druck $p$ :

$$
F(p, \rho, \vartheta ิ)=0,
$$

wie z.B. bei idealen Gasen

$$
p \cdot V=m \cdot \boldsymbol{R}_{G} \cdot \text { ข. }
$$

Hierbei bedeutet $V$ das Volumen, $m=\rho \cdot V$ die Gasmasse, $R_{G}$ die spezifische Gaskonstante und $\vartheta$ die Temperatur. Damit stehen die grundlegenden Gleichungen für die Bewegung der idealen Fluide fest; die Frage nach dem Strömungszustand führt auf Anfangsund Randwertprobleme. Zur Untersuchung der instationären Strömung in der Filter-Cigarette bzw. in der Rauchmaschine wird dieser "direkte Lösungsweg" ver- lassen und verallgemeinerte dynamische Fluid-Netzwerkelemente für das Meßsystem (Beobachtungssystem) "Fluidströmung in Rohrleitungen" bzw. Rohrschüttungen eingeführt. In (1) Abbildung 23 ist die Entwicklung eines mathematischen Modells, z.B. der dynamischen Strömungsvorgänge in einer Filter-Cigarette, als eine abstrahierende Folge von Abbildungen dargestellt:

\section{PROZESS \\ $\Downarrow$ \\ MEß- \\ MODELL MATHEMATISCHES \\ SYSTEM $\Rightarrow$ SYSTEM $\Rightarrow \quad$ SYSTEM}

Für die Druckangaben wird

$p \quad:$ Absolutdruckangabe

$P \quad:$ Unterdruck bezüglich der Atmosphäre $p_{\text {atm }}$

$$
P=p_{\text {atm }}-p
$$

vereinbart und die Pfeilrichtungen entsprechend ((1), Abbildung 3), gewählt.

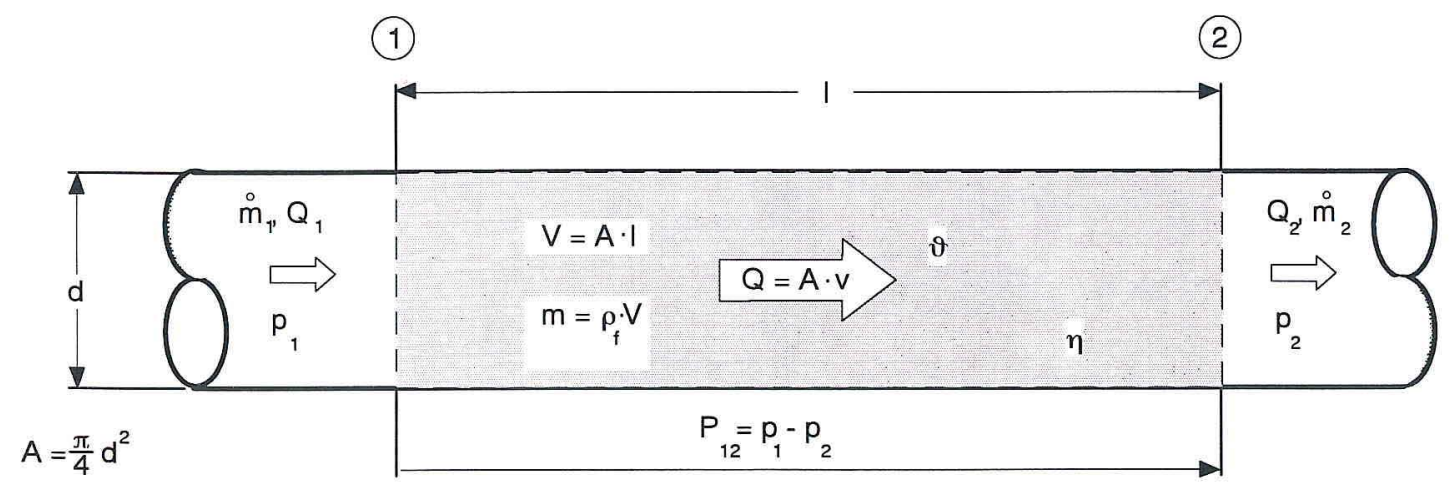

Bezugsdruck Vakuum: $\mathrm{p}=0$ 


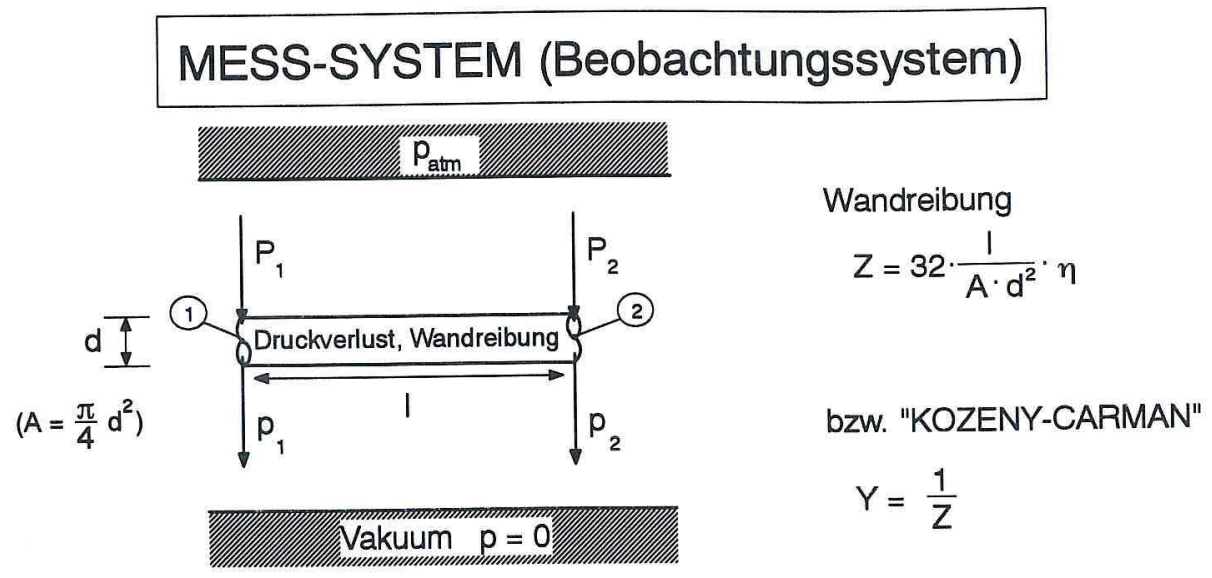

\section{MODELL-SYSTEM (Fluid-Netzwerkelement)}

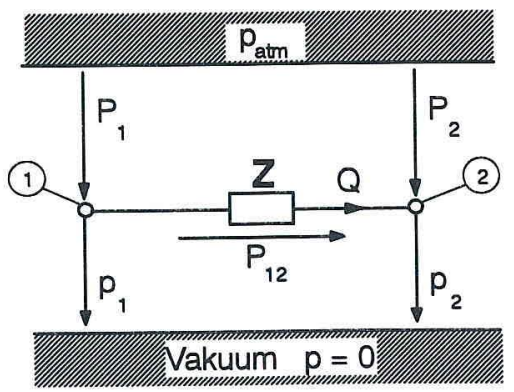

$$
\begin{aligned}
P & :=p_{a t m}-p \\
P_{12} & =p_{1}-p_{2} \\
& =P_{2}-P_{1}
\end{aligned}
$$

\section{MATHEMATISCHES-SYSTEM}

a) Strukturbild (Signalflußdiagramm)

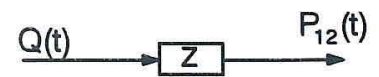

$$
\text { URSACHE }
$$

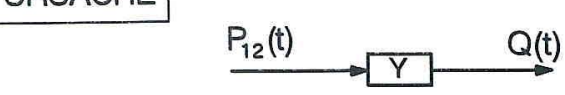

WIRKUNG

b) Mathematische Systembeschreibung

$$
\begin{aligned}
& P_{12}:=Z \cdot Q \\
& Q:=Y \cdot P_{12}
\end{aligned}
$$

Abbildung 4.

Die Strömungs-Impedanz $Z=1 / Y$ (Druckverlustdarstellung $P=p_{\text {atm }}-p((1)$, Abbildung 3). 


\subsection{Strömungsimpedanz Z}

Es wird eine laminare Strömung vorausgesetzt. Zwischen Volumenstrom $Q$ und Druckverlust $P$ besteht eine lineare Beziehung; die Proportionalitätskonstante $Z$ wird Strömungsimpedanz genannt:

$$
P_{12}=Z \cdot Q \text {. }
$$

Damit lassen sich stationäre Fluid-Netzwerke vollständig beschreiben. Grundlage ist die stationäre BERNOULLI-Gleichung als einfache Druckverlustbeziehung für einen Rohrleitungsabschnitt (Abbildung 3) mit

$$
Z=32 \cdot \frac{1}{A \cdot d^{2}} \cdot \eta,
$$

bzw. für eine Rohrschüttung wie bei einem Cigarettenstrang gilt die KOZENY-CARMAN-Gleichung (1) mit

$$
\begin{aligned}
Z & =K \cdot \frac{l_{S}}{A} \cdot S_{m}^{2} \cdot \eta \frac{\rho_{p}^{2}}{\left(1-\left(\frac{\rho_{p}}{\rho_{T}}\right)\right)^{3}} \\
& =K \cdot \frac{l_{S}}{A} \cdot S_{m}^{2} \cdot \eta \cdot \rho_{T}^{2} \cdot \frac{(1-\epsilon)^{2}}{\epsilon^{3}}
\end{aligned}
$$

Hierbei bedeuten:

$P_{12}:$ Druckverlust ("Zugwiderstand = pressure drop")

$K$ : Konstante

$l_{s} \quad$ : Stranglänge

$S_{n}$ : Spezifische Oberfläche

$\rho_{p}:$ Packungsdichte $=$ Stopfdichte

$\rho_{T}:$ Tabakdichte

$\rho_{f}:$ Dichte des Gases (Fluid)

$\eta \quad$ : dynamische Viskosität des Gases

$\varepsilon \quad:$ Porosität (Porenvolumen, 4, 5, 6)

Q : Durchfluß

A : Querschnittsfläche

d : Durchmesser

Der Kehrwert $Y=1 / Z$ ist die Strömungsadmittanz. Abbildung 4 zeigt die symbolische Darstellung als verallgemeinertes Fluid-Netzwerkelement mit dem Bezugsdruck Vakuum $p=0$ (Absolutdruck $p$ ) und Atmosphäre $p_{\text {arm }}$ (Unterdruck $P$ ). Der Druckverlust $P_{12}$ und damit indirekt auch die Strömungsimpedanz $Z$ sind durch Messung einfach zu ermitteln.

\subsection{Fluid-Induktivität $L$}

Für die Bewegung der Fluidmasse im Strömungsfaden bzw. in einem Rohr, das vollständig von dem Fluid ausgefüllt ist (Kolbenströmung, plug flow model), gilt aufgrund des NEWTONschen Grundgesetzes [3.1] (Druckkraft $P_{12} \cdot \mathrm{A}$, Abbildung 3)

$$
P_{12} \cdot A=m \cdot \frac{\mathrm{d} v}{\mathrm{~d} t} .
$$

\section{MESS-SYSTEM (Beobachtungssystem)}

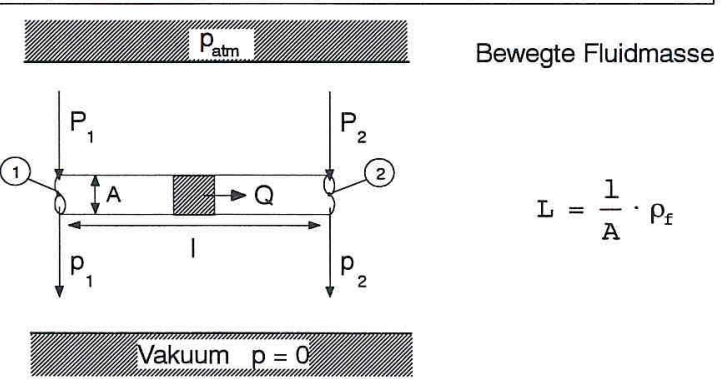

\section{MODELL-SYSTEM (Fluid-Netzwerkelement)}

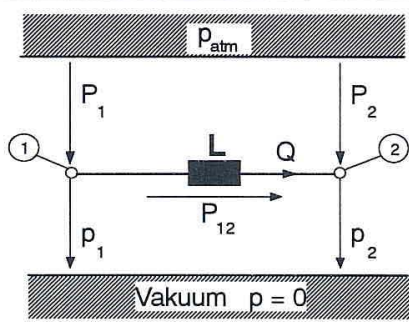

$$
\begin{aligned}
P & :=p_{a t m}-p \\
P_{12} & =p_{1}-P_{2} \\
& =P_{2}-P_{1}
\end{aligned}
$$

\section{MATHEMATISCHES-SYSTEM}

a) Strukturbild (Signalflußdiagramm)

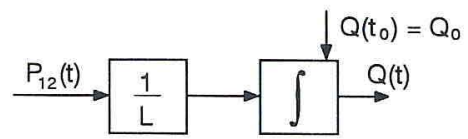

b) Mathematische Systembeschreibung

$$
\begin{aligned}
& \frac{d Q}{d t}=\frac{1}{L} \cdot P_{12}, Q\left(t_{0}\right)=Q_{0} \\
& \text { bzW. } \\
& Q(t)=Q_{0}+\int_{t_{0}}^{t} \frac{1}{L} \cdot P_{12}(\tau) d \tau
\end{aligned}
$$

Abbilldung 5.

Fluid-Induktivität $L$ (Druckverlustdarstellung $P=p_{\text {atm }}-p$ ). 
Mit der Fluidmasse $m=V \cdot \rho_{f}$ und der Fluidströmung $Q=A \cdot v$ erhält man eine Differentialgleichung 1 . Ordnung

$$
\frac{\mathrm{d} Q}{\mathrm{~d} t}=\frac{1}{L} \cdot P_{12}, Q\left(t_{0}\right)=Q_{0},
$$

wobei die verallgemeinerte Fluid-Induktivität

$$
L=\frac{1}{A} \cdot \rho_{f}
$$

ein kinetische Energie speicherndes Element ist. Gleichung [3.11] entspricht dem instationären Anteil in der BERNOULLIschen Gleichung [3.2]. Die Darstellung als Netzwerkelement bzw. als MATHEMATISCHES SYSTEM zeigt Abbildung 5.

Für eine Rohrschüttung (Tabakstrang, Cigarette) gilt

$$
L=\frac{l_{f}}{A_{f}} \cdot \rho_{f}
$$

mit

$l_{f}$ : "mittlere Fluid-Strömungslänge"

$A_{f}$ : "mittlerer Fluid-Strömungsquerschnitt"

$V:$ Gesamtvolumen $(\mathrm{V}=\mathrm{A} \cdot \mathrm{l})$

$V_{f}:$ Fluidvolumen $\left(V_{f}=A_{f} \cdot l_{f}\right)$

$V_{T}:$ Tabakvolumen $\left(V=V_{f}+V_{T}\right)$.

Nach Einführung der Porosität $\varepsilon$ einer Schüttung (Lückengrad, spezifisches Hohlraumvolumen; $(4,5,6)$ )

$$
\begin{aligned}
\varepsilon & =\frac{\text { Fluidvolumen }}{\text { Gesamtvolumen }} \\
& =\frac{V_{f}}{V}=1-\frac{V_{T}}{V}=1-\frac{\rho_{p}}{\rho_{T}}
\end{aligned}
$$

läßt sich die Fluidinduktivität abschätzen, z.B. mit $l_{f}$ $\approx l$ folgt

$$
L=\frac{1}{A} \cdot \frac{\rho_{f}}{\varepsilon}=\frac{1}{A} \cdot \frac{\rho_{f}}{1-\frac{\rho_{P}}{\rho_{T}}}
$$

Ist die Packungsdichte (Stopfdichte) $\rho_{p} \approx 270 \mathrm{mg} / \mathrm{ml}$ und die Tabakdichte $\rho_{T} \approx 1400 \mathrm{mg} / \mathrm{ml}$, so beträgt die Porosität $\varepsilon \approx 0,8$. Das zu betrachtende Fluidvolumen ist demnach $80 \%$ vom Tabakstrang-Gesamtvolumen.

\subsection{Fluidkapazität C}

Im stationären Fall genügt ein Fluid der Masse $\mathrm{m}=$ $\rho_{f} \cdot V$, das sich in einem Speicher mit dem Volumen
$V$ befindet, der Zustandsgleichung [3.5]. Bei dynamischen Änderungen gilt für das Beobachtungssystem (MEßSYSTEM, Abbildung 3) die Massenbilanz

$$
\frac{\mathrm{d}}{\mathrm{d} t}\left(\frac{p \cdot V}{\boldsymbol{R}_{G} \cdot \vartheta}\right)=\stackrel{\circ}{\mathrm{v}}_{1}-\stackrel{\circ}{2}_{2}
$$

hierbei sind $m_{1}=\rho_{\mathrm{fl}} \cdot Q_{1}$ und

$$
m_{2}=\rho_{\mathrm{f} 2} \cdot Q_{2}
$$

die zu- und abfließenden Massenströme.

Bei isothermer Kompression $(\vartheta=$ const.), geringen Dichteschwankungen $\left(\rho_{\mathrm{f}}=\rho_{\mathrm{f} 1}=\rho_{\mathrm{f} 2}\right)$ und konstantem Volumen erhält man eine Differentialgleichung 1 . Ordnung

$$
\frac{\mathrm{d} p}{\mathrm{~d} t}=\frac{1}{C} \cdot\left(Q_{1}-Q_{2}\right), p\left(t_{0}\right)=p_{0},
$$

mit der verallgemeinerten Fluid-Kapazität

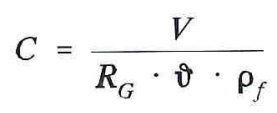

als ein Speicherlement der potentiellen Energie (Abbildung 6).

Für eine Rohrschüttung folgt entsprechend zur FluidInduktivität

$$
C=\frac{V_{f}}{R_{G} \cdot \vartheta} \cdot \frac{1}{\rho_{f}}=\frac{V}{R_{G} \cdot \vartheta} \cdot \frac{\varepsilon}{\rho_{f}}
$$

\subsection{Kenngrößen einer Filter-Cigarette}

Eine Übersicht zur Modellbildung mit Hilfe physikalischer Analogien, hier elektrische und strömungsmechanische, zeigt Abbildung 7.

Für die fiktive Filter-Cigarette im King-Size-Format seien ergänzend folgende Daten zum kanonischen Zahlenbeispiel gegeben:

$$
\begin{aligned}
& l_{S}=63 \mathrm{~mm} \quad \text { Tabakstranglänge } \\
& l_{M}=84 \mathrm{~mm} \quad \text { Cigarettenlänge } \\
& d=7,95 \mathrm{~mm} \quad \text { Durchmesser } \\
& A=49,64 \mathrm{~mm}^{2} \quad \text { Querschnitt }\left(d^{2} \cdot \pi / 4\right) \\
& V_{C i g}=4,17 \mathrm{~cm}^{3} \quad \text { Cigarettenvolumen }\left(l_{M} \cdot A\right) \\
& \varepsilon=0,8 \quad \text { Porosität } \\
& \rho_{f}=1,293 \mathrm{kgm}^{-3} \quad \text { Luftdichte (Fluid) } \\
& \vartheta=295 \mathrm{~K} \quad \text { Fluidtemperatur }\left(22{ }^{\circ} \mathrm{C}\right) \\
& R_{G}=285 \mathrm{~m}^{2} \mathrm{~s}^{-2} \mathrm{~K}^{-1} \text { spezifische Gaskonstante für } \\
& \text { Luft }
\end{aligned}
$$

daraus folgt für die gesamte Filter-Cigarette 
$\mathrm{Z}_{\mathrm{M}}=68,57 \cdot 10^{6} \mathrm{~Pa} \cdot \mathrm{s} \cdot \mathrm{m}^{-3}=6,857 \mathrm{daPa} \cdot \mathrm{s}^{\cdot} \cdot \mathrm{ml}^{-1}$

Strömungsimpedanz infolge Strömungswiderstand; Druckverlustmessung [2.3], (Abbildung 1 und 2),

$L_{M}=1,75 \cdot 10^{3} \mathrm{~kg} \cdot \mathrm{m}^{-4}=1,7510^{-6} \mathrm{~kg} \cdot \mathrm{ml}^{-1} \cdot \mathrm{mm}^{-1}$ Strömungsinduktivität $\left(\mathrm{l}_{\mathrm{M}} / \mathrm{A}\right) \cdot\left(\rho_{\mathrm{f}} / \varepsilon\right)$ infolge Fluidmasse,

$$
\begin{aligned}
C_{M} & =0,0307 \cdot 10^{-9} \mathrm{~m}^{4} \cdot \mathrm{s}^{2} \cdot \mathrm{kg}^{-1} \\
& =30,7 \cdot 10^{-3} \mathrm{ml} \cdot \mathrm{mm} \cdot \mathrm{s}^{2} \cdot \mathrm{kg}^{-1}
\end{aligned}
$$

Strömungskapazität $\left(V_{C i g} /\left[R_{G} \cdot \vartheta\right]\right) \cdot\left(\varepsilon / \rho_{\mathrm{f}}\right)$ infolge Volumenspeicher.

\section{MESS-SYSTEM (Beobachtungssystem)}

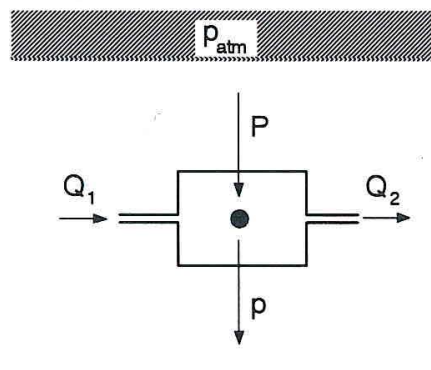

Fluid-Volumenspeicher

Vakuum $\mathrm{p}=0$

\section{MODELL-SYSTEM (Fluid-Netzwerkelement)}
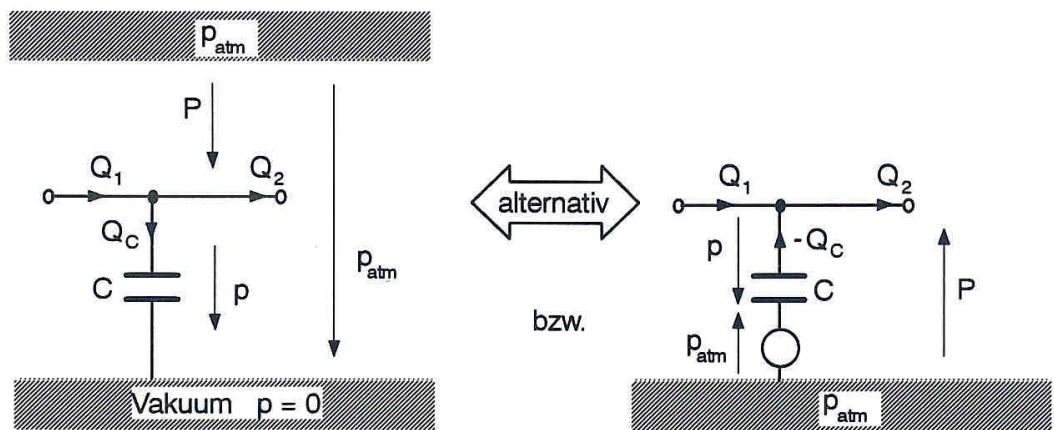

\section{MATHEMATISCHES-SYSTEM}

a) Strukturbild (Signalflußdiagramm)

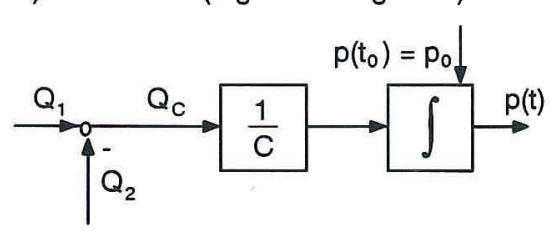

b) Mathematische Systembeschreibung

$$
\begin{gathered}
\frac{d p}{d t}=\frac{1}{C} Q_{C} \\
p\left(t_{0}\right)=p_{0}
\end{gathered}
$$

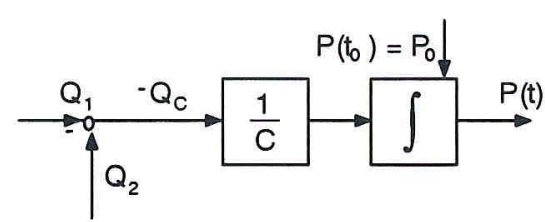

$$
\begin{aligned}
& \frac{d P}{d t}=-\frac{1}{C} Q_{C} \\
& P\left(t_{0}\right)=P_{0}=P_{a t m}-P_{0}
\end{aligned}
$$




\begin{tabular}{|c|c|}
\hline Elektrisch & FLUID MECHANIK \\
\hline Widerstand & Strömungsimpedanz \\
\hline$\stackrel{\mathrm{i}_{\mathrm{R}} \mathrm{U}_{1}}{\underset{\mathrm{U}_{\mathrm{R}=\mathrm{U}_{1}-\mathrm{U}_{2}}}{\mathrm{R}=\mathrm{G}^{-1}}} \mathrm{U}_{2}$ & 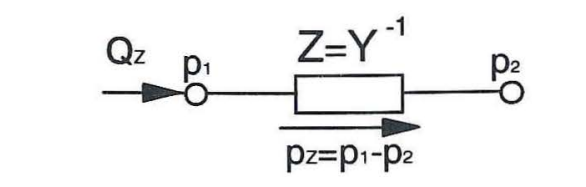 \\
\hline$U_{R}=R i_{R}$ & $\mathrm{pz}=Z \mathrm{Qz}$ \\
\hline Kapazität & Fluid-Kapazität \\
\hline$\stackrel{\mathrm{i}_{\mathrm{c}}}{\longrightarrow} \underset{\mathrm{U}}{\stackrel{\mathrm{U}_{\mathrm{c}}=\mathrm{U}_{1}-\mathrm{U}_{2}}{\longrightarrow}} \stackrel{\mathrm{C}}{\mathrm{U}}$ & 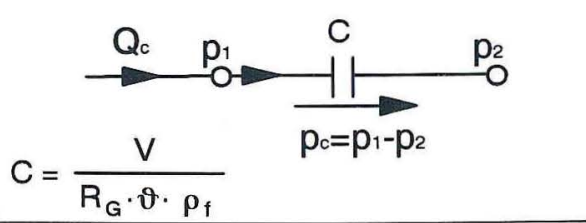 \\
\hline$\frac{d u_{c}}{d t}=\frac{1}{C} \quad i_{c}$ & $\frac{d p_{c}}{d t}=\frac{1}{C} Q_{c}$ \\
\hline Induktivität & Fluid-Induktivität \\
\hline$\stackrel{i}{\longrightarrow} U_{O} \rightarrow \underset{U_{\mathrm{L}=\mathrm{U}_{1}-\mathrm{U}_{2}}}{\mathrm{~L}} \mathrm{U}_{2}$ & 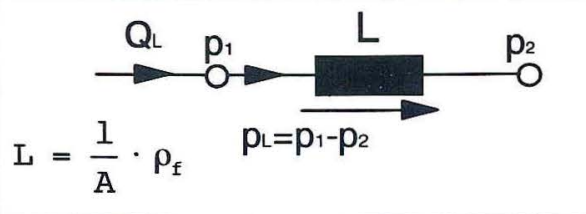 \\
\hline$\frac{d i L}{d t}=\frac{1}{L} U_{L}$ & $\frac{\mathrm{dQ}_{\mathrm{L}}}{\mathrm{dt}}=\frac{1}{\mathrm{~L}} \mathrm{pL}$ \\
\hline $\begin{array}{ll}\sum i_{v}=0 & \text { 1. Kirchhoff. Gesetz } \\
\sum u_{v}=0 & \text { 2. Kirchhoff. Gesetz }\end{array}$ & $\begin{array}{ll}\sum_{v} Q_{v}=0 & \text { Knotenregel } \\
\sum_{v} p_{v}=0 & \text { Maschenregel }\end{array}$ \\
\hline $\begin{array}{ll}{[\mathrm{C}]=\mathrm{F}} & \text { Kapazität } \\
{[\mathrm{L}]=\mathrm{H}} & \text { Induktivität } \\
{[\mathrm{R}]=\Omega} & \text { Widerstand } \\
{[\mathrm{G}]=\Omega^{-1}} & \text { Leitwert } \\
{[\mathrm{u}]=\mathrm{V}} & \text { Spannung } \\
{[\mathrm{i}]=\mathrm{A}} & \text { Strom }\end{array}$ & $\begin{array}{ll}{[\mathrm{C}]=\mathrm{m}^{4} \mathrm{~s}^{2} \mathrm{~kg}^{-1}} & \text { "Fluidvolumen" } \\
{[\mathrm{L}]=\mathrm{kg} \mathrm{m}^{-4} \text { "Fluidmasse" }} \\
{[\mathrm{Z}]=\mathrm{Pa} \mathrm{s} \mathrm{m}^{-3} \quad \text { Impedanz }} \\
\mathrm{Y}]=\mathrm{m}^{3} \mathrm{~s}^{-1} \mathrm{~Pa}^{-1} & \text { Admittanz } \\
{[\mathrm{p}]=\mathrm{Pa}} & \text { Druck } \\
{[\mathrm{Q}]=\mathrm{m}^{3} \mathrm{~s}^{-1}} & \text { Volumenstrom }\end{array}$ \\
\hline
\end{tabular}


Gemeinsames Merkmal der Systemparameter $Z, L$ und $C$ ist ihre Zeitinvarianz. So erfolgt die Umformung von [3.16] in [3.17] nur unter der Bedingung eines konstanten Volumens.
Bei der Abrauchmaschine wird mittels einer Kolbenpumpe ein Unterdruck erzeugt, indem das Hubvolumen stetig vergrößert wird, und daher ist ein zeitvariables Volumen $V(t)$ zu berücksichtigen (Abbildung 8).

\section{MESS-SYSTEM (Beobachtungssystem)}

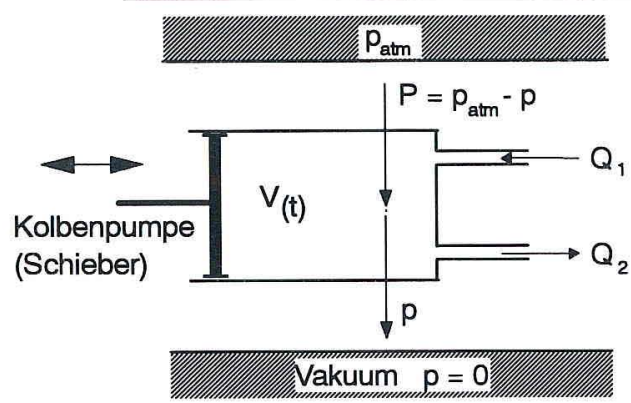

$$
\begin{aligned}
& C(t)=\frac{V(t)}{R_{G} \cdot \vartheta \cdot \rho_{f}} \\
& \dot{C}=\frac{1}{R_{G} \cdot \vartheta \cdot \rho_{f}} \cdot \frac{d V(t)}{d t} \\
& Q_{C}=Q_{1}-Q_{2}
\end{aligned}
$$

\section{MODELL-SYSTEM (Fluid-Netzwerkelement)}

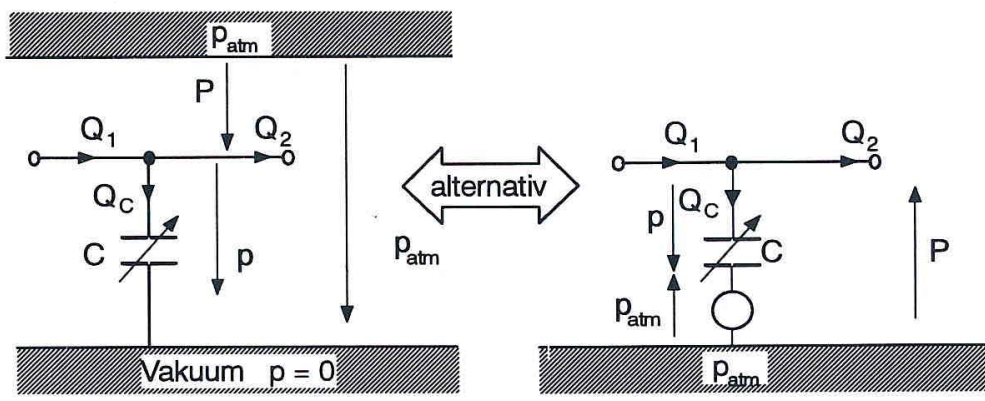

\section{MATHEMATISCHES-SYSTEM}

a) Strukturbild (Signalflußdiagramm)

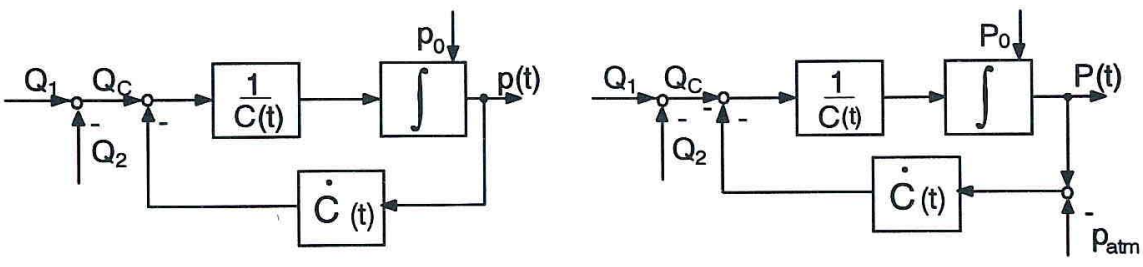

b) Mathematische Systembeschreibung

$$
\begin{aligned}
& \frac{d p}{d t}=-\frac{\dot{C}}{C} \cdot p+\frac{1}{C} \cdot Q_{C} \quad ; \quad \frac{d P}{d t}=-\frac{\dot{C}}{C} \cdot\left(P-p_{a t m}\right)-\frac{1}{C} \cdot Q_{c} \\
& \mathrm{p}\left(\mathrm{t}_{0}\right)=\mathrm{p}_{0} \quad \mathrm{P}\left(\mathrm{t}_{0}\right)=\mathrm{P}_{0}=\mathrm{p}_{\mathrm{atm}}-\mathrm{p}_{0}
\end{aligned}
$$

$$
p(t)=p_{0}+\int_{t_{0}}^{t}\left\{-\frac{C(\tau)}{C(\tau)} \cdot p(\tau)+\frac{1}{C(\tau)} \cdot Q_{C}(\tau)\right\} d \tau
$$


Mit der Produktregel der Differentialrechnung

$$
\begin{gathered}
\frac{\mathrm{d}}{\mathrm{d} t}\left(\frac{p(t) \cdot V(t)}{R_{G} \cdot \vartheta}\right) \\
=\frac{\mathrm{d} p(t)}{\mathrm{d} t} \cdot \frac{V(t)}{R_{G} \cdot \hat{\vartheta}}+\frac{p(t)}{R_{G} \cdot \hat{v}} \cdot \frac{\mathrm{d} V(t)}{\mathrm{d} t},
\end{gathered}
$$

der zeitvarianten Fluidkapazität

$$
C(t)=\frac{V(t)}{\boldsymbol{R}_{G} \cdot \hat{U} \cdot \rho_{f}}
$$

und der Kapazitätsänderung

$$
\dot{C}(t)=\frac{\mathrm{d} C}{\mathrm{~d} t}=\frac{1}{\boldsymbol{R}_{G} \cdot \boldsymbol{v} \cdot \rho_{f}} \cdot \frac{\mathrm{d} V(t)}{\mathrm{d} t}
$$

folgt aus der Massenbilanz [3.16]

$$
\begin{gathered}
\frac{\mathrm{d} p(t)}{\mathrm{d} t}=\frac{\dot{C}(t)}{C(t)} \cdot p(t) \\
+\frac{1}{C(t)} \cdot\left(Q_{1}(t)-Q_{2}(t)\right) \\
p\left(t_{0}\right)=p_{0}
\end{gathered}
$$

eine Differentialgleichung 1. Ordnung mit zeitvarianten Koeffizienten.

In der Unterdruckdarstellung lautet die Differentialgleichung für ein zeitvariantes Fluidvolumen mit $P=$ $p_{\text {arm }}-p$ und $\mathrm{d} P / \mathrm{d} t=-\mathrm{d} p / \mathrm{d} t$

$$
\begin{gathered}
\frac{\mathrm{d} P(t)}{\mathrm{d} t}=\frac{\dot{C}(t)}{C(t)} \cdot\left(P(t)-p_{\mathrm{atm}}\right)-\frac{1}{C(t)} \cdot\left(Q_{1}(t)-Q_{\dot{t}}\right. \\
P\left(t_{0}\right)=p_{\mathrm{atm}}-p_{0} .
\end{gathered}
$$

\section{DIE FILTER-CIGARETTE ALS DYNAMISCHES SYSTEM}

\subsection{Dynamisches Modell mit verteilten Parametern}

Es wird die Ortsabhängigkeit von Druckverlust und Strömung im homogenen Tabakstrang behandelt. "Homogen" soll bedeuten, daß Strangstücke gleicher Länge auch gleiche Parameter $Z_{1}^{\prime}, L_{1}^{\prime}, Y_{1}^{\prime}$ und $C_{1}^{\prime}$ haben. Dabei nennt man die konstanten verteilten Parameter:
$Z_{1}^{\prime} \quad$ : Strömungs-Impedanzbelag (Abbildung 1),

$L^{\prime} \quad$ : Strömungs-Induktivitätsbelag

$$
\left(L^{\prime}=\frac{1}{A} \cdot \frac{\rho_{f}}{\varepsilon}\right),
$$

$Y_{2}^{\prime} \quad$ : Strömungs-Admittanzbelag (Abbildung 1)

$$
\left(Y_{2}^{\prime}=\frac{1}{Z_{2}^{\prime}}\right),
$$

$C^{\prime} \quad$ : Strömungs-Kapazitätsbelag

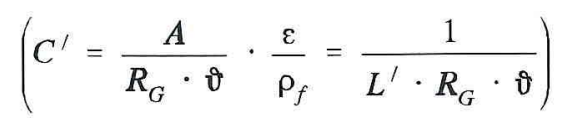

Um die Abhängigkeit des Volumenstroms $Q(x, t)$ und des Druckverlustes $P(x, t)$ von der Ortskoordinate $x$ und der Zeit $t$ abzuleiten, genügt es, die Maschenund Knotenregel wie in (1) heranzuziehen und auf den Strangabschnitt der Länge $\delta x$ entsprechend Abbildung $9 \mathrm{~b}$ anzuwenden. Man erhält dann die beiden verkoppelten linearen partiellen Differentialgleichungen mit konstanten Koeffizienten

$$
\begin{aligned}
& \frac{\delta P}{\delta x}=Z_{1}^{\prime} \cdot Q+L^{\prime} \cdot \frac{\mathrm{d} Q}{\mathrm{~d} t} \\
& \frac{\delta Q}{\delta x}=Y_{2}^{\prime} \cdot P+C^{\prime} \cdot \frac{\mathrm{d} P}{\mathrm{~d} t},
\end{aligned}
$$

oder eine gleichwertige lineare partielle Differentialgleichung 2. Ordnung, die Telegrafengleichung

$$
\begin{gathered}
\frac{\delta^{2} P}{\delta x^{2}}=Z_{1}^{\prime} \cdot Y_{2}^{\prime} \\
\cdot P+\left(Z_{1}^{\prime} \cdot C^{\prime}+Y_{2}^{\prime} \cdot L^{\prime}\right) \cdot \frac{\mathrm{d} P}{\mathrm{~d} t} \\
+L^{\prime} \cdot C^{\prime} \cdot \frac{\mathrm{d}^{2} P}{\mathrm{~d} t^{2}} .
\end{gathered}
$$

Eine allgemeine analytische Lösung der Gleichungen [4.1] und [4.2] bzw. [4.3] für beliebige Anfangs- und Randwertbedingungen existiert nicht. Man muß sich darauf beschränken, gewisse Spezialfälle zu behandeln. In der Leitungstheorie ist dies der Betrieb mit sinusförmigen Zeitfunktionen, z.B. in der komplexen Schreibweise

$$
(j=\sqrt{-1}):
$$




$$
\begin{gathered}
P(x, t)=\boldsymbol{P}(x) \cdot \cos (\omega t) \\
=\operatorname{Re}\left\{\boldsymbol{P}(x) \cdot \mathrm{e}^{\mathrm{j} \omega t}\right\} .
\end{gathered}
$$

Ein Ansatz $P_{(x)}=P_{0} \cdot \mathrm{e}^{\gamma x}$ führt dann auf die Lösung

$$
P(x)=P_{E} \cdot \operatorname{ch}(\gamma x)+Q_{E} \cdot Z_{W} \cdot \operatorname{sh}(\gamma x)
$$

$$
Q(x)=\frac{P_{E}}{Z_{W}} \cdot \operatorname{sh}(\gamma x)+Q_{E} \cdot \operatorname{ch}(\gamma x)
$$

mit der "komplexen" Fortpflanzungskonstante

$$
\gamma=\sqrt{\left(Z_{1}^{\prime}+j \omega L^{\prime}\right) \cdot\left(Y_{2}^{\prime}+j \omega C^{\prime}\right)}
$$

und dem "komplexen" Wellenwiderstand

$$
Z_{W}=\sqrt{\frac{\left(Z_{1}^{\prime}+j \omega L^{\prime}\right)}{\left(Y_{2}^{\prime}+j \omega C^{\prime}\right)}}
$$

als eine verallgemeinerte Darstellung zu dem stationären Strömungsfall in (1), Gleichung [4.12], [4.13]). Die Lösung und Analyse im Frequenzbereich mit Wechselströmungsgrößen wurde in einem Vortrag anläßlich des 36. Tabak-Kolloquiums in Lübeck, 5. Juni bis 10. Juni 1994, vorgestellt (Zugprofil-Fluiddynamik im Zeit-und Spektralbereich).

(a)

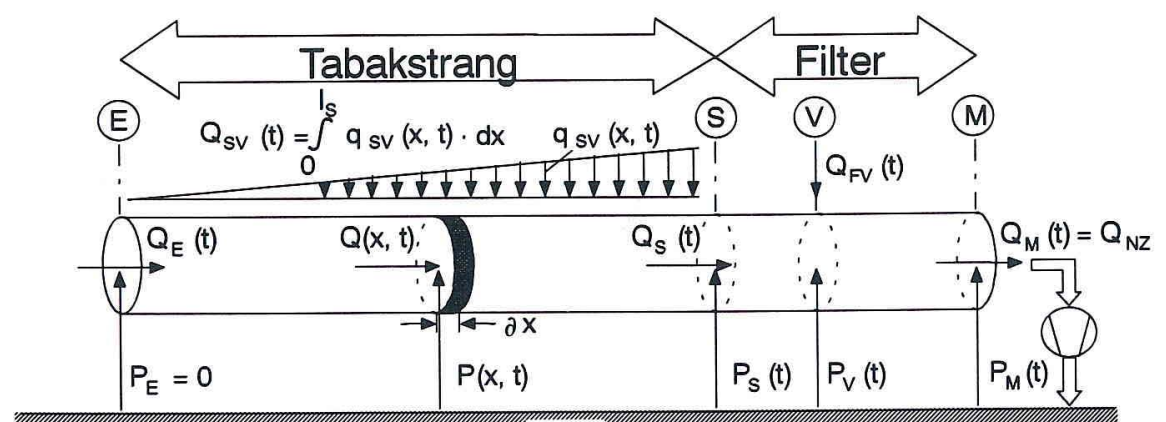

(b)

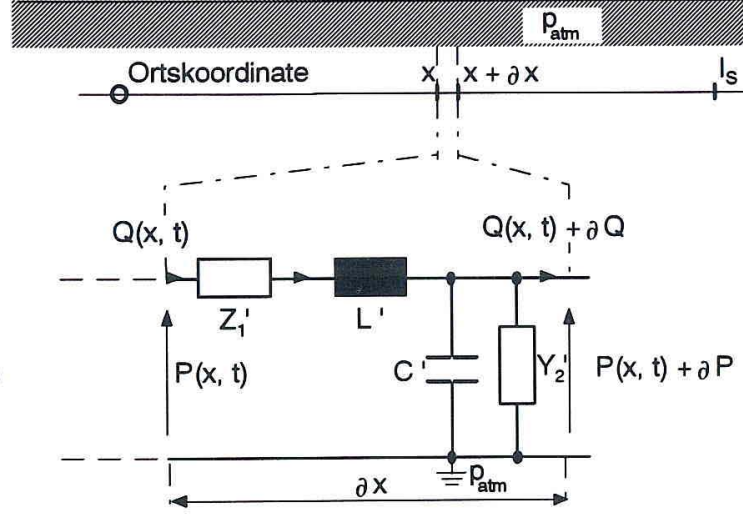

(c)

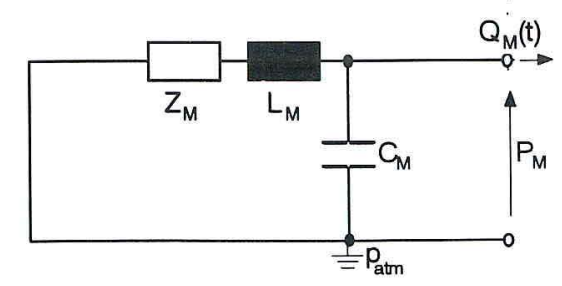

\section{Abbildung 9.}

Dynamisches Modell einer Filter-Cigarette;
a) Wirkschaltbild (MEBSYSTEM)
b) Fluidnetzwerk mit verteilten Fluidparametern
$\left[Z_{1}^{\prime}, L^{\prime}, C^{\prime}, Y_{2}^{\prime}\right]$ (MODELLSYSTEM)
c) einfaches dynamisches Netzwerk mit konzentrierten Parametern (MODELLSYSTEM). 


\subsection{Elementares dynamisches Strömungsmodell mit} konzentrierten Parametern

Alternativ zu dem dynamischen verteilten ParameterModell soll nun ein sehr einfaches elementares dynamisches Modell mit konzentrierten Parametern betrachtet werden (Abbildung 9c, 10).

Der PROZESS ist "die instationäre Strömung in einer
Filter-Cigarette". Das MEßSYSTEM (Beobachtungssystem) ist die Filter-Cigarette mit den globalen Parametern

$\begin{array}{ll}\text { Druckverlust } & \Rightarrow Z_{M} \\ \text { Fluidmasse } & \Rightarrow L_{M} \\ \text { Volumenspeicher: } & \Rightarrow C_{M} .\end{array}$

\section{MESS-SYSTEM (Beobachtungssystem)}

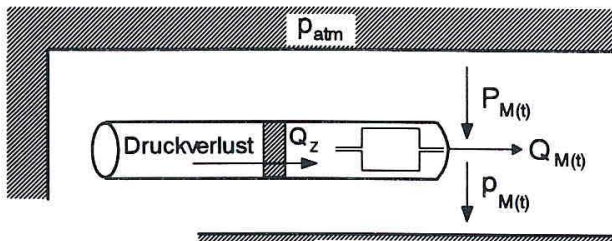

Vakuum $\mathrm{p}=\mathrm{O}=\mathrm{M}$

\section{MODELL-SYSTEM (Fluid-Netzwerkelement)}

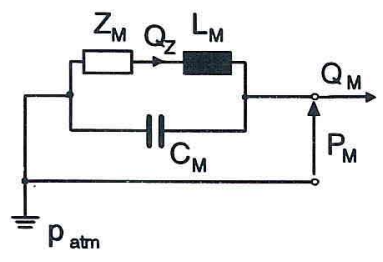

(Vernachlässigung der Fluidmasse)

$$
L_{M} \rightarrow 0
$$

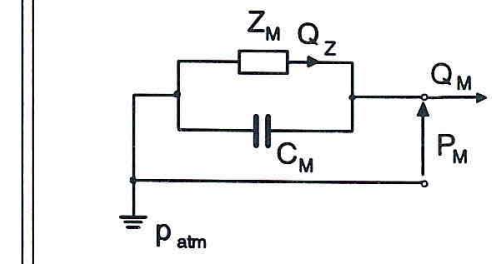

\section{MATHEMATISCHES-SYSTEM}

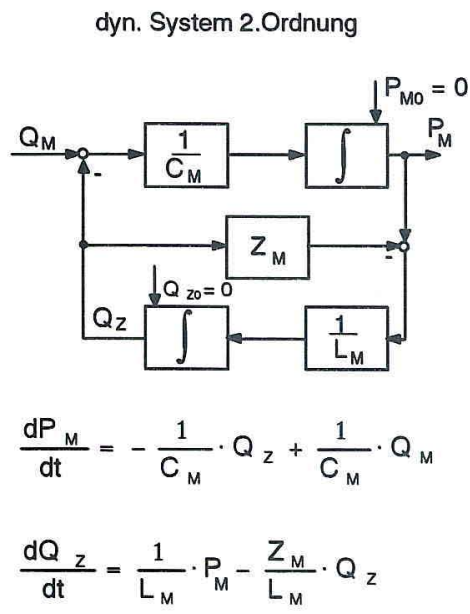

dyn. System 1.Ordnung

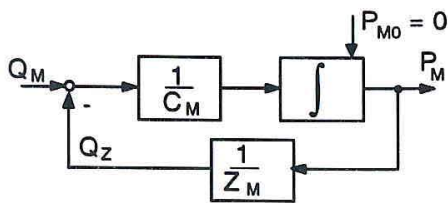

$\frac{d P_{M}}{d t}=-\frac{1}{Z_{M} \cdot C_{M}} \cdot P_{M}+\frac{1}{C_{M}} \cdot Q_{M}$

$0=P_{M}-Z_{M} \cdot Q_{Z}$

Abbildung 10.

Elementares dynamisches Strömungsmodell einer Filter-Cigarette mit konzentrierten Parameter $\left[Z_{M}, C_{M}, L_{M}\right]$. 
Zum Zeitpunkt $t_{0}=0$ ist die Strömung in der FilterCigarette in Ruhe, d.h. der Unterdruck $P_{M 1}\left(t_{0}\right)=P_{M O}=$ 0 und die Strömung $Q_{Z}\left(t_{0}\right)=Q_{Z 0}=0$.

Die speziellen Fluidelemente im MEßSYSTEM ersetzt man durch die aus Abbildung 4, 5 und 6 bekannten Netzwerkelemente und gewinnt so das MODELL-SYSTEM, ein dynamisches Fluidnetzwerk. Die topologische Zuordnung der Elemente ergibt sich, wenn man die an den Elementen wirksamen Größen Druck und Volumenstrom berücksichtigt.

Das MATHEMATISCHE SYSTEM läßt sich sehr einfach mit den bewährten Methoden der elektrischen Netzwerkanalyse entwickeln und unmittelbar in einem Strukturbild (Signalflußdiagramm) darstellen. Daraus sind dann die mathematischen Gleichungen einfach ablesbar.

Als Ergebnis erhält man ein System von zwei linearen Differentialgleichungen 1. Ordnung mit konstanten diskreten Parametern. Eine allgemeine analytische Lösung kann mit herkömmlichen Methoden bestimmt werden.

Kann die bewegte Fluidmasse vernachlässigt werden, folgt ein sehr einfaches dynamisches System 1. Ordnung.

Wird die LAPLACE-Transformation $\left(P_{M}(\mathrm{~s})=\mathscr{L}\left\{P_{M}(t)\right\}\right.$, $\left.Q_{M}(s)=\mathscr{L}\left\{Q_{M}(t)\right\}\right)$ auf das dynamische System im Zeitbereich $t$ (Strukturbild bzw. Differentialgleichung) angewendet, liefert diese im sogenannten Bildbereich $s$ die Eingangs-Ausgangs-Beschreibung

$$
P_{M}(s)=G(s) \cdot Q_{M}(s)
$$

mit der Übertragungsfunktion $G(s)$ für das dynamische System 2. Ordnung

$$
G_{2}(s)=Z_{M} \cdot \frac{1+\frac{L_{M}}{Z_{M}} \cdot s}{C_{M} \cdot L_{M} \cdot\left(s^{2}+\frac{Z_{M}}{L_{M}} \cdot s+\frac{1}{C_{M} \cdot L_{M}}\right)}
$$

bzw. für das dynamische System 1. Ordnung (Fluidmasse vernachlässigt, d.h. $L_{M} \rightarrow 0$ )

$$
G_{1}(s)=Z_{M} \cdot \frac{1}{C_{M} \cdot Z_{M} \cdot\left(s+\frac{1}{C_{M} \cdot Z_{M}}\right)}
$$

Die Eigenwerte $\mathrm{s}_{\mathrm{i}}$ (Eigenschwingung $\sim \mathrm{e}^{\mathrm{si} \cdot{ }^{\mathrm{t}}}$ ) des dynamischen Systems sind die Nullstellen des sogenannten charakteristischen Polynoms bzw. des (ungekürzten) Nennerpolynoms der Übertragungsfunktion.

Bei dem dynamischen System 2. Ordnung folgen aus

$$
s^{2}+\frac{Z_{M}}{L_{M}} \cdot s+\frac{1}{C_{M} \cdot L_{M}}=0
$$

die Eigenwerte $\mathrm{S}_{\mathrm{i}}$

$$
\begin{aligned}
s_{i} & =-\frac{Z_{M}}{2 \cdot L_{M}} \pm \sqrt{\left(\frac{Z_{M}}{2 L_{M}}\right)^{2}-\frac{1}{C_{M} \cdot L_{M}}} \\
& =\frac{Z_{M}}{L_{M}}\left(-\frac{1}{2} \pm \frac{1}{2} \sqrt{1-\frac{4 \cdot L_{M}}{Z_{M}^{2} \cdot C_{M}}}\right) \\
& \approx \frac{Z_{M}}{L_{M}}\left(-\frac{1}{2} \pm\left(\frac{1}{2}-\frac{L_{M}}{Z_{M}^{2} \cdot C_{M}}\right)\right),
\end{aligned}
$$

mit der Näherungsbeziehung wegen

$$
L_{M} \ll Z_{M}^{2} \cdot C_{M} .
$$

Für das kanonische Zahlenbeispiel folgt damit

$$
\begin{aligned}
s_{c}= & -0,4809 \cdot 10^{3} \cdot \frac{1}{s} \approx-\frac{1}{Z_{M} \cdot C_{M}} \\
s_{L} & =-38,702 \cdot 10^{3} \cdot \frac{1}{s} \\
& \approx-\frac{Z_{M}}{L_{M}}+\frac{1}{Z_{M} \cdot C_{M}} \approx-\frac{Z_{M}}{L_{M}} .
\end{aligned}
$$

Die Zeitkonstanten $T_{i}=-1 / s_{i}$ der Eigenschwingungen $\mathrm{e}^{-\mathrm{t} / T_{1}}$ sind dann $\left(T_{C} \approx Z_{M} \cdot C_{M}, T_{L} \approx L_{M} / Z_{M}\right)$

$$
T_{C}=2,0793 \mathrm{~ms}
$$

$$
T_{L}=0,0258 \mathrm{~ms}
$$

Das dynamische Verhalten ist in Abbildung 11 dargestellt.

Eine Eigenschwingung infolge der Fluidinduktivität (bewegte Fluidmasse) ist vernachlässigbar $\left(T_{L}<<T_{C}\right)$ und ein dynamisches System 1. Ordnung zur Beschreibung der instationären Fluidströmung ausreichend. Charakteristische Kenngröße des dynamischen Systems 1. Ordnung ist die Zeitkonstante $T_{C}$. Sie ist abhängig von Zugwiderstand und Volumenspeicher 

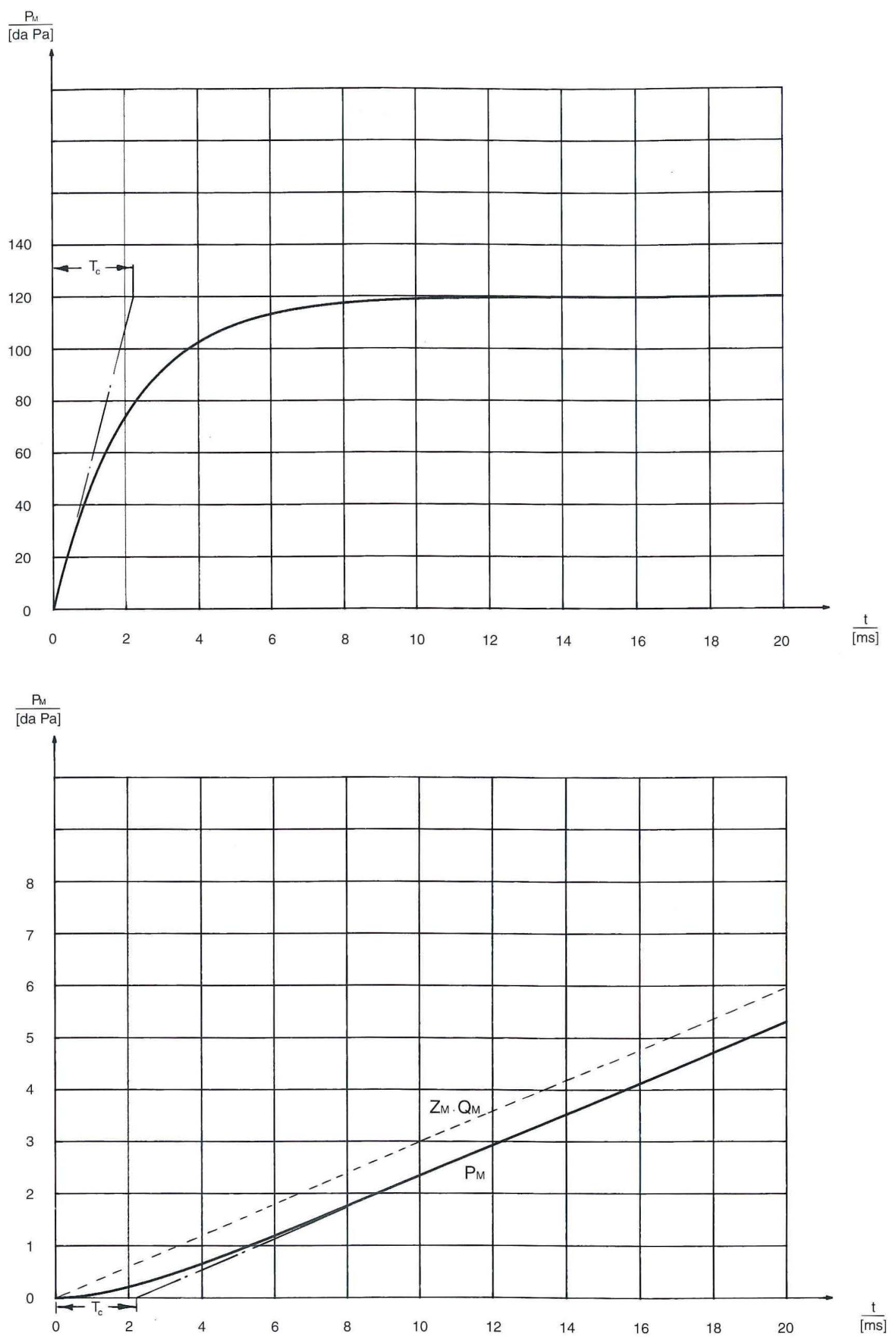

\section{Abbildung 11.}

Beschreibung der instationären Strömungsvorgänge in einer Filter-Cigarette mit einem dynamischen System 2. Ordnung.

a) Sprungantwort für Eingangsgröße $Q_{M}$

$Q_{M}(t)=17,5 \mathrm{ml} / \mathrm{s}$ für $\mathrm{t}>0$,
b) Rampenantwort für Eingangsgröße $Q_{M}$

$Q_{M}(t)=\alpha \cdot t\left(\alpha=17,5 \cdot(\pi / 2)^{2} \mathrm{~m} / / \mathrm{s}^{2}\right)$. 

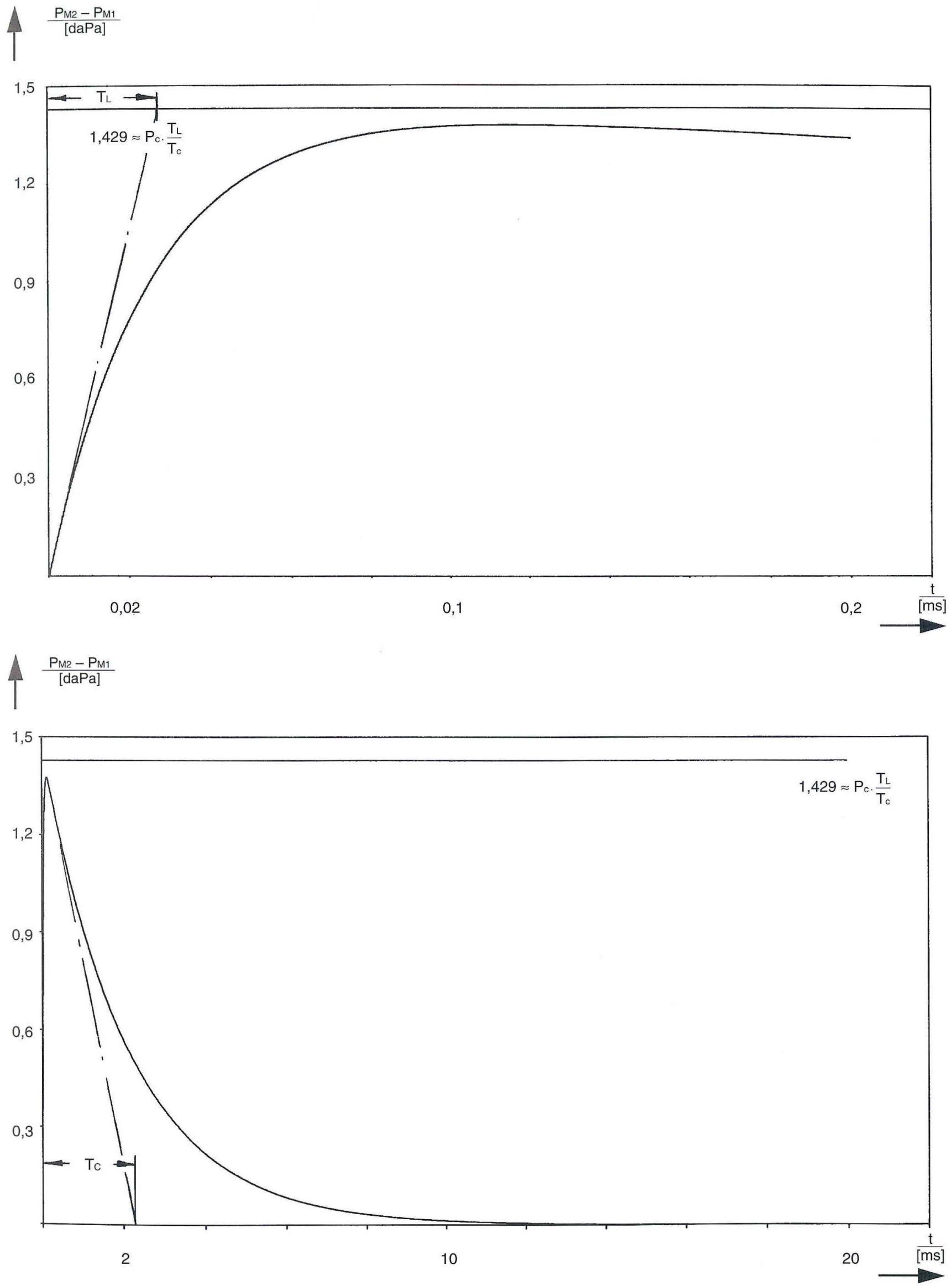

Abbildung 12.

$\left(P_{M 2}(t)-P_{M-1}(t)\right)$ : Druckverlust-Abweichung in der Sprungantwort bei einem dynamischen System 1. Ordnung $\left(P_{M 1}(t)\right)$ und dem 2. Ordnung $\left(P_{M 2}(t)\right)$. 


$$
T_{C}=Z_{M} \cdot C_{M} \approx 2 \mathrm{~ms} .
$$

Das dynamische Verhalten für die Eingangsgröße $Q_{M}(t)$ ist entsprechend Abbildung 11. Für den Druckverlust $P_{M}(t)$ ist die Abweichung in der Sprungantwort zwischen dem dynamischen System 1.Ordnung $P_{M 1}(t)$ und dem dynamischen System 2. Ordnung $P_{\lambda 12}(t)$ (Abb. 12)

$$
\begin{aligned}
P_{M 2}(t)-P_{M I}(t)= & P_{c} \cdot \frac{1}{2} \cdot\left(1-\sqrt{1-\frac{4 \cdot L_{M}}{Z_{M}^{2} \cdot C_{M}}}\right) \\
& \cdot\left(e^{-\frac{t}{T_{c}}}-e^{-\frac{t}{T_{L}}}\right)
\end{aligned}
$$

mit der Amplitude

$$
\begin{aligned}
1,4729 \text { daPa } & =P_{c} \cdot \frac{1}{2} \cdot\left(1-\sqrt{1-\frac{4 \cdot L_{M}}{Z_{M}^{2} \cdot C_{M}}}\right) \\
& \approx P_{c} \cdot \frac{T_{L}}{T_{c}} .
\end{aligned}
$$

Die konstanten Parameter sind einfach zu ermitteln, die Ersatzströmungsimpedanz $Z_{M}$ durch Druckverlustmessung der offenen "kalten" Cigarette [2.3] bzw. über die KOZENY-CARMAN-Gleichung [3.9] und die Ersatzströmungskapazität $C_{M}$ infolge Volumenspeicher mit [3.19].

Eine Modellierung der Cigarette mit konzentrierten Parametern ist hinreichend, das dynamische System 1. bzw. 2. Ordnung läßt sich analytisch allgemein für beliebige Eingangsgrößen elementar lösen und durch signifikante Zeitkonstanten $T_{C} \approx 2 \mathrm{~ms}$ bzw. $T_{C}$ und $T_{L}$ $\approx 0,02 \mathrm{~ms}$ charakterisieren.

Die zeitliche Verzögerung des Zugprofils in der Abrauchmaschine mit einer Systemzeitkonstante von ca. $T=50$ bis $100 \mathrm{~ms}$ liegt wesentlich über der dominierenden Zeitkonstante der Filter-Cigarette mit $T_{C}=2$ ms; Ursache für dieses träge Eigenverhalten ist das System "Abrauchmaschine".

\section{DIE CIGARETTE IN DER ABRAUCHMA- SCHINE}

\subsection{Prozeß - physikalisches System}

Als PROZESS bzw. physikalisches System wird die instationäre Fluidströmung in der Abrauchmaschine betrachtet.

\subsection{Meßsystem - Beobachtungssystem}

Der Prozeß ist nur über seine erfaßbaren Größen, seine Daten und das a priori Wissen wahrnehmbar, dies ist das Meßsystem bzw. das Beobachtungssystem.

DIN/ISO 3308 (2) beschreibt beispielhaft die besonderen Merkmale einer gängigen Abrauchmaschine, die mit einem Zugmechanismus nach dem Kolbenpumpenprinzip ausgestattet ist (Abbildung 13).

Zum Zeitpunkt $t_{0}=0$ wird mittels einer Kolbenpumpe ein Unterdruck $P_{M}$ an die Cigarette angelegt. Der daraus resultierende Volumenstrom $Q_{M}$ am Mundstück der Cigarette verändert sich so, daß sich ein glockenförmiges Zugprofil ergibt.

Nach Überschreiten eines Maximums $Q_{M m}$ zum Zeitpunkt $t_{n}$ vermindert sich der Volumenstrom $Q_{M}$ während der weiteren Zugdauer, bis er den Wert $Q_{M d}$ zum Zeitpunkt $t_{d}$, der Zugzeit, erreicht, an dem die Saugvorrichtung keinen weiteren Unterdruck mehr erzeugt, aber noch ein Druckunterschied im System vorhanden ist. Danach vermindert sich der Volumenstrom $Q_{M}$ asymptotisch auf den Wert Null, der zum Zeitpunkt $t_{e}$ erreicht wird. Theoretisch erfolgt dies nach unendlich langer Zeit, praktisch wird diese Zeit $\mathrm{t}_{e}$ durch die Standard-Zugfrequenz begrenzt.

Das Totvolumen $V_{T}$ ist das Volumen zwischen dem Mundstück $M$ einer Cigarette und der Saugvorrichtung; dieses muß so klein wie möglich sein und darf $100 \mathrm{ml}$ nicht überschreiten.

Das gesamte Hubvolumen $V_{H g}$ ist das Volumen der verdrängten Luft zwischen dem oberen und unteren Totpunkt des Kolbens; in der Zeit zwischen $t_{0}=0$ : $V_{H}\left(t_{0}\right)=0$ und der Zugzeit $t_{d}: V_{H}\left(t_{d}\right)=V_{H_{g}}$ beträgt es $V_{H}(t)$, d.h. $t_{0} \leq t \leq t_{d}: 0 \leq V_{H}(t) \leq V_{H g}$.

Das Zugvolumen $V_{M}$ ist das Fluidvolumen, das aus dem Mundstückende einer Cigarette tritt und hat mit dem Zugprofil, dem Volumenstrom $Q_{M}$, der direkt hinter dem Mundstückende einer Cigarette gemessen wird, die Relation

$$
\begin{gathered}
V_{M}(t)=\int_{t_{0}=0}^{t} Q_{M}(\tau) \mathrm{d} \tau \quad \text { bzw. } \\
Q_{M}(t)=\frac{\mathrm{d} V_{M}(t)}{\mathrm{d} t} .
\end{gathered}
$$

Ohne Cigarette (Strömungsimpedanz Null) erhält man ein ideales Zugprofil, das sogenannte Hubvolumen- 

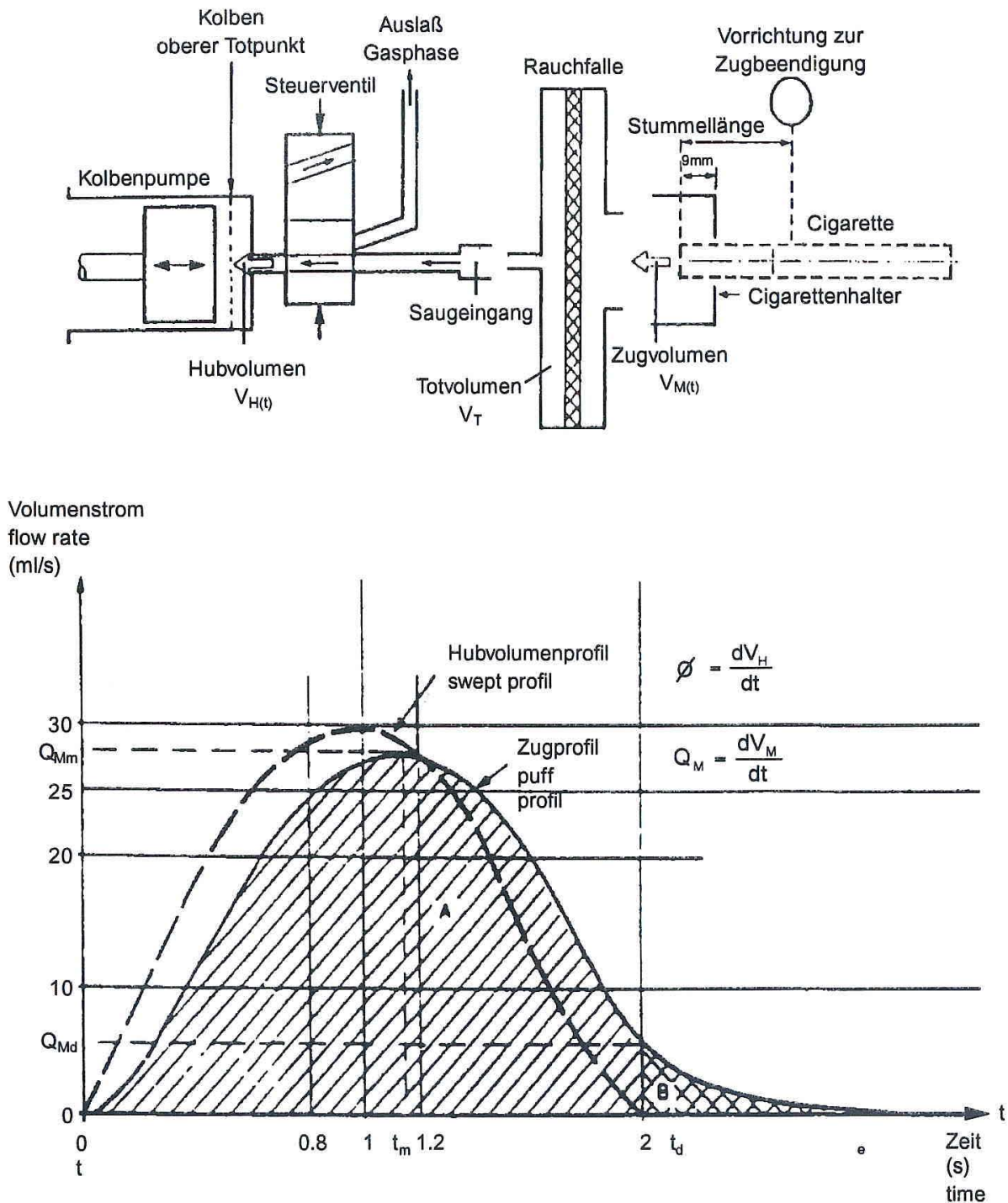

Abbildung 13.

MEBSYSTEM (Beobachtungssystem): Schematische Darstellung einer Abrauchmaschine mit Cigarette und eines Zugprofils (2).

profil $\phi$, und es gilt die Beziehung

$$
\phi(t)=\frac{\mathrm{d} V_{H}(t)}{\mathrm{d} t}
$$

$$
V_{H}(t)=V_{H g} \cdot \frac{1}{2} \cdot\left(1-\cos \left(\pi \cdot \frac{t}{t_{\mathrm{d}}}\right)\right)
$$

bzw. für das Hubvolumenprofil

Bei herkömmlichen Kolben- / Kurbelwellenmechanismen (2) folgt aus der Kurbelwelle-Pleuelstangen-Geometrie für das Hubvolumen

$$
\Phi(t)=\frac{V_{H g}}{t_{\mathrm{d}}} \cdot \frac{\pi}{2} \cdot \sin \left(\pi \cdot \frac{t}{t_{\mathrm{d}}}\right)
$$




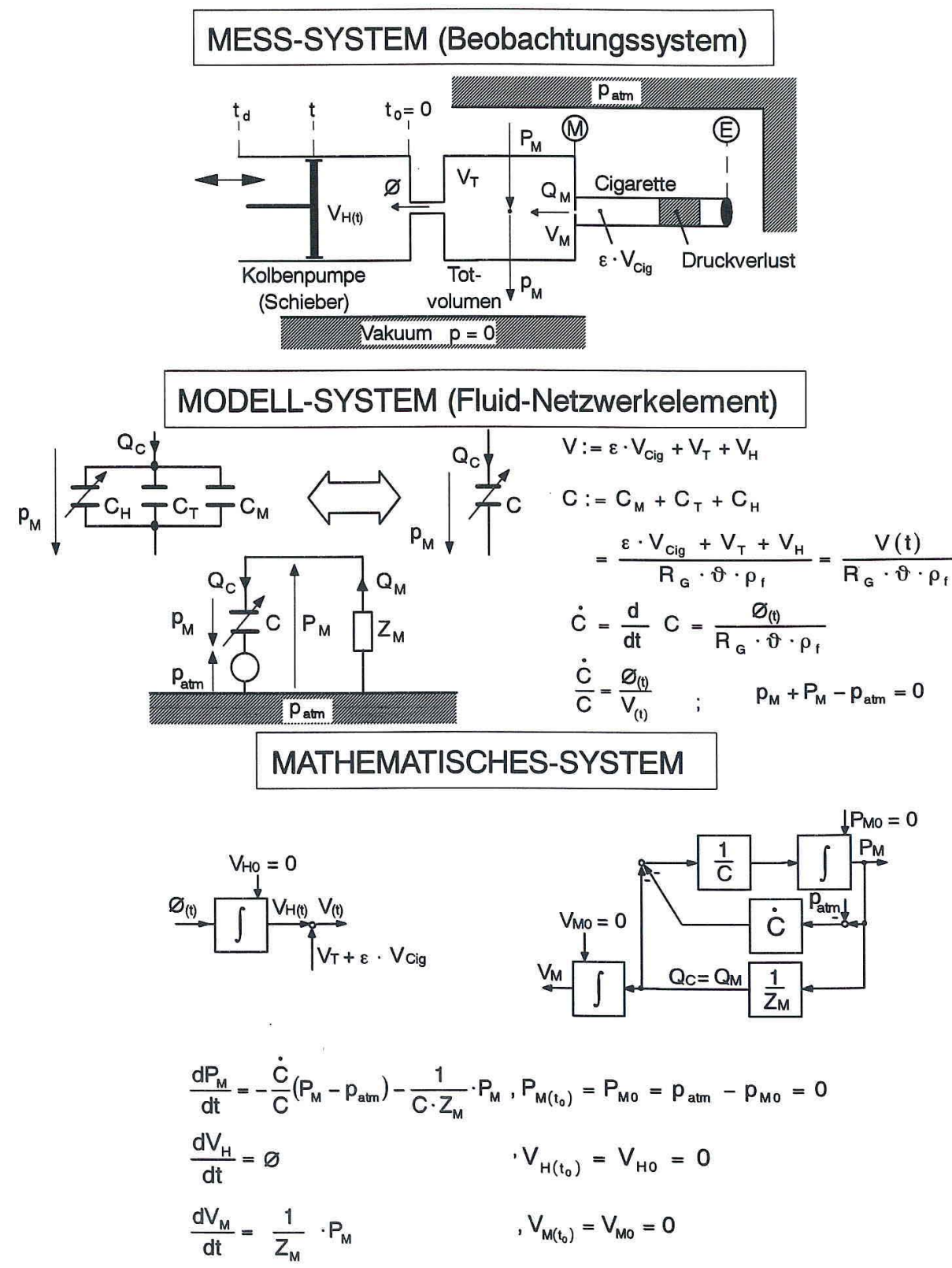

Abbilldung 14.

Dynamisches Strömungsmodell einer Cigarette in der Abrauchmaschine.

Das Hubvolumenprofil hat zum Zeitpunkt $t_{0}=0$ für ein kleines Zeitintervall $0 \leq t \leq 0,1 \cdot t_{\mathrm{d}}$ den zeitlichen Verlauf einer Rampenfunktion (Tangente bei $t_{0}=0$ )

$$
\phi(t)=\frac{V_{H g}}{2} \cdot\left(\frac{\pi}{t_{\mathrm{d}}}\right)^{2} \cdot t,
$$

für $0 \leq t \leq 0,1 \cdot t_{d}$.

Übliche Zahlenwerte sind:

$$
\begin{aligned}
V_{H g} & =35 \cdot 10^{-6} \mathrm{~m}^{3}=35 \mathrm{ml} \\
t^{T} & \leq 100 \cdot 10^{-6} \mathrm{~m}^{3}=100 \mathrm{ml} \\
t_{d} & =2 \mathrm{~s} \\
t_{c} & =60 \mathrm{~s}
\end{aligned}
$$

Damit ist die Entwicklung des MEßSYSTEMs abgeschlossen und mit den Elementen der Fluiddynamik erhält man Abbildung 14a. 


\subsection{Modellsystem}

Das MODELLSYSTEM, in Form eines dynamischen Fluidnetzwerkes, gewinnt man durch einfaches Ersetzen der speziellen Fluidelemente durch die bekannten Netzwerkelemente, insbesondere durch die zeitvariable Fluidkapazität $C_{H}(t)$ um das zeitabhängige Hubvolumen $V_{H}(t)$ zu berücksichtigen (Abbildung 14b).

Das Gesamtvolumen ist die Summe aller Teilvolumina

$$
V(t)=\varepsilon \cdot V_{C i g}+V_{T}+V_{H}(t)
$$

und mit der Definitionsgleichung für die Fluidkapazität [3.18] erhält man für die gesamte Fluidkapazität die bekannte Tatsache, daß Parallelschaltungen von (Fluid-) Kapazitäten sich addieren

$$
\begin{aligned}
& C(t)=C_{M}+C_{T}+C_{H}(t) \\
& =\frac{\varepsilon \cdot V_{C i g}+V_{T}+V_{H}(t)}{R_{G} \cdot \hat{v} \cdot \rho_{f}} \\
& =\frac{V(t)}{R_{G} \cdot \vartheta \cdot \rho_{f}} .
\end{aligned}
$$

Mit der Fluidkapazität $C(t)$ folgt ein sehr einfaches Fluidnetzwerk (Abbildung 14b).

\subsection{Mathematisches System}

Das MATHEMATISCHE SYSTEM läßt sich in einem Strukturbild darstellen und daraus das nichtlineare dynamische System 3. Ordnung mit diskreten zeitvariablen Koeffizienten ablesen. Eingangsgrößen sind das Hubvolumenprofil $\phi(t)$ und der (konstante) Atmosphärendruck $p_{\mathrm{atm}}=1,01325 \cdot 10^{5} \mathrm{~Pa}(1,01325 \mathrm{bar}=$ 1 atm).

Eine allgemeine analytische Lösung ist hier aus zwei Gründen erschwert, dies sind die zeitvariablen Parameter und die Nichtlinearität. Daher ist eine Charakterisierung mit einer Zeitkonstanten $T$ nicht zulässig. Zur groben Abschätzung der Dynamik von CigaretteRauchmaschine läßt sich eine Zeitkonstante

$$
\begin{gathered}
\left(\frac{C}{\dot{C}}=\frac{V}{\phi} \geq 5 s>C \cdot Z_{m}\right) \\
T_{C R}=C \cdot Z_{M}
\end{gathered}
$$

bzw. $\left(P_{c}\right.$ Druckverlust der offenen Cigarette [2.3], $V=V_{H}+V_{T}+\varepsilon \cdot V_{C i g}$

$$
\frac{T_{C R}}{[m s]}=0,055 \cdot \frac{V}{[m l]} \cdot \frac{P_{C}}{[d a P a]}
$$

aus Abb. 14 ablesen, man erhält mit

$$
\begin{gathered}
V_{T}+\varepsilon \cdot V_{C i g}=100 \mathrm{ml}, 0 \leq V_{H}(t) \leq 35 \mathrm{ml} \\
\text { und } P_{C}=120 \mathrm{daPa}
\end{gathered}
$$

$$
66 \mathrm{~ms} \leq T_{C R} \leq 88,5 \mathrm{~ms}
$$

Im Sinne von "Ursache $\rightarrow$ Wirkung" sind die instationären fluidmechanischen Vorgänge anhand des Netzwerkes und des Signalflußplanes deutlich zu erkennen und erlauben einen tiefen Einblick in die innere Struktur des Systems.
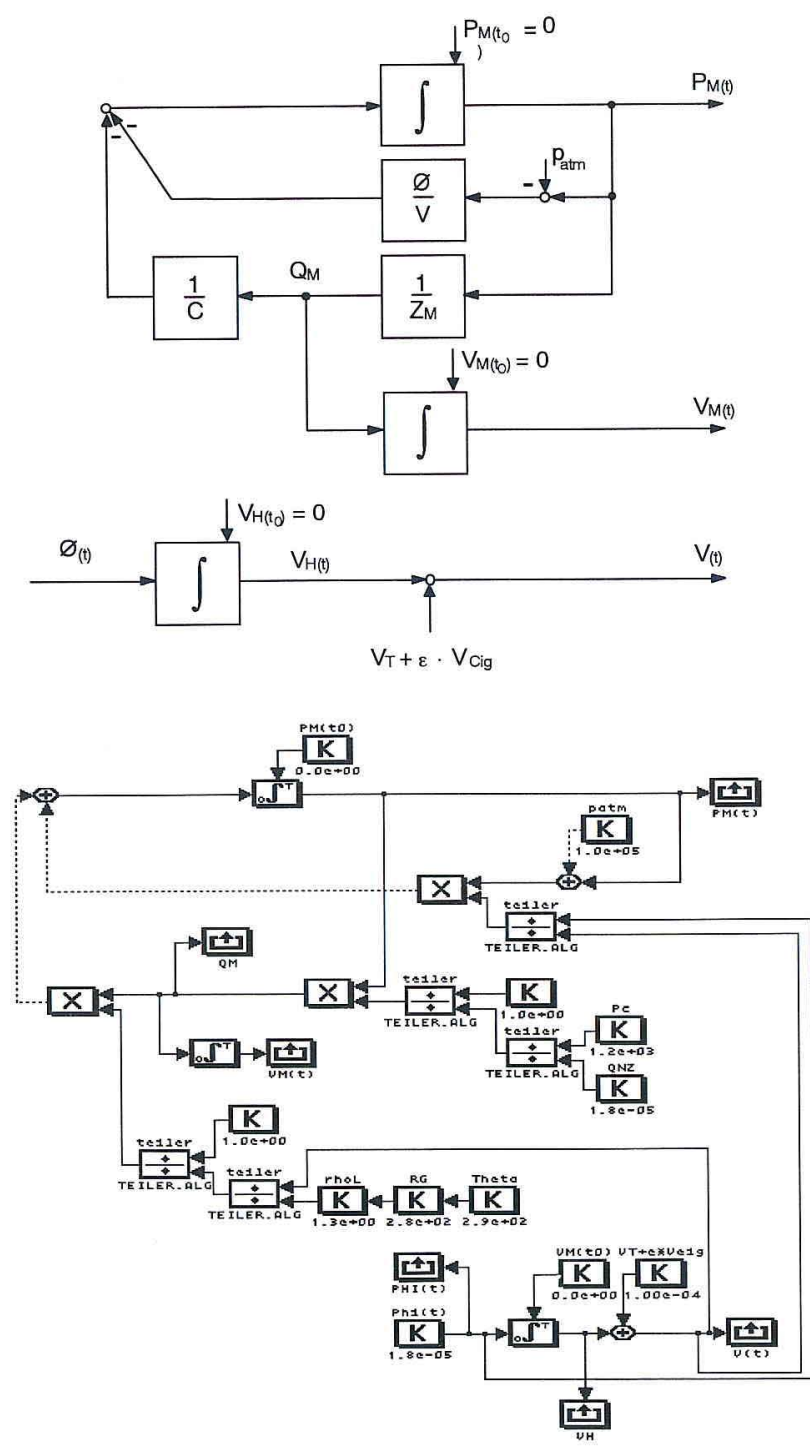

Abbildung 15.

Modifiziertes dynamisches Strömungsmodell einer Cigarette in einer Abrauchmaschine a) und blockorientierte grafische Konfiguration zur Simulation mittels XANALOG b). 


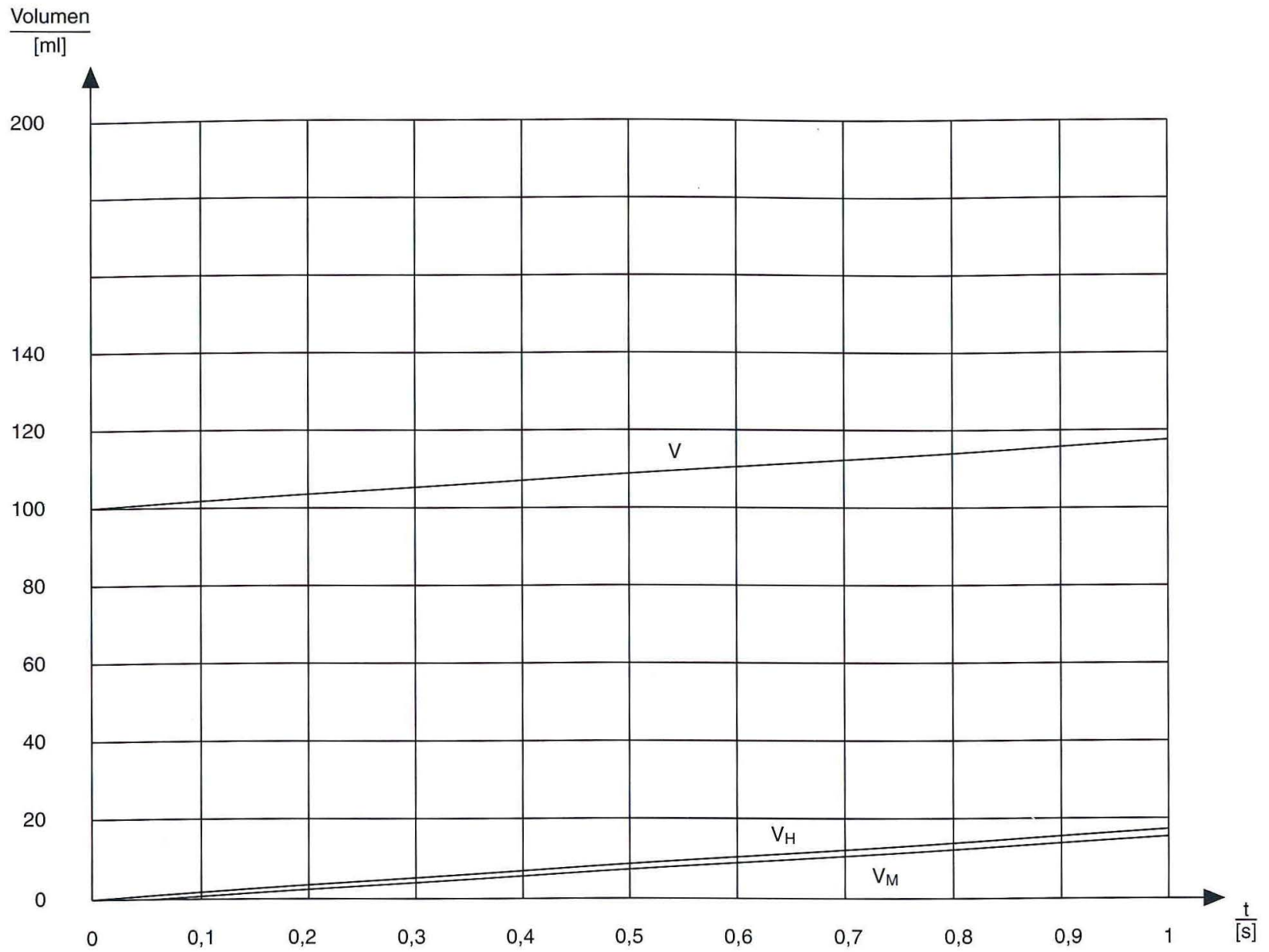

\section{Volumenstrom}

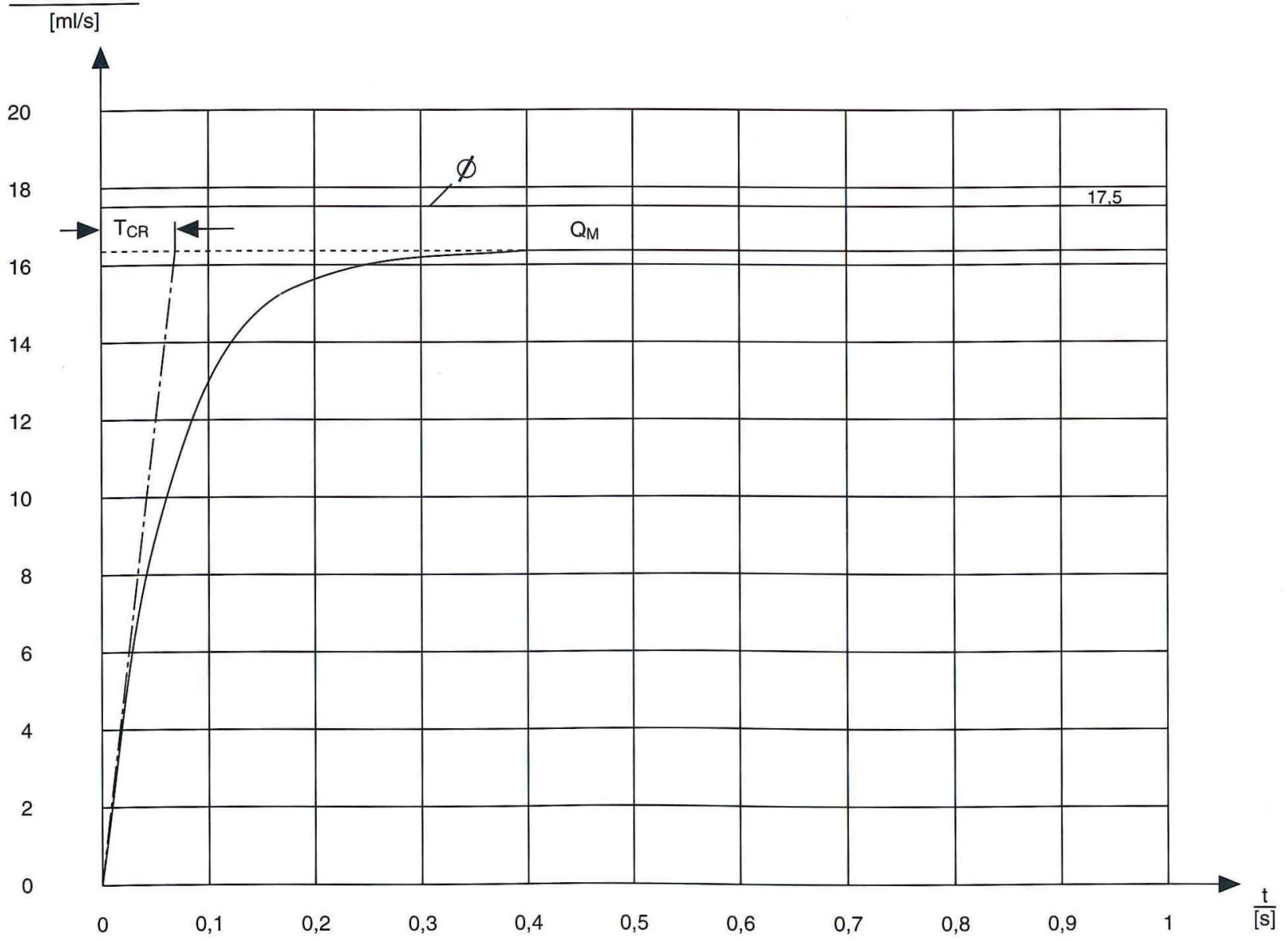




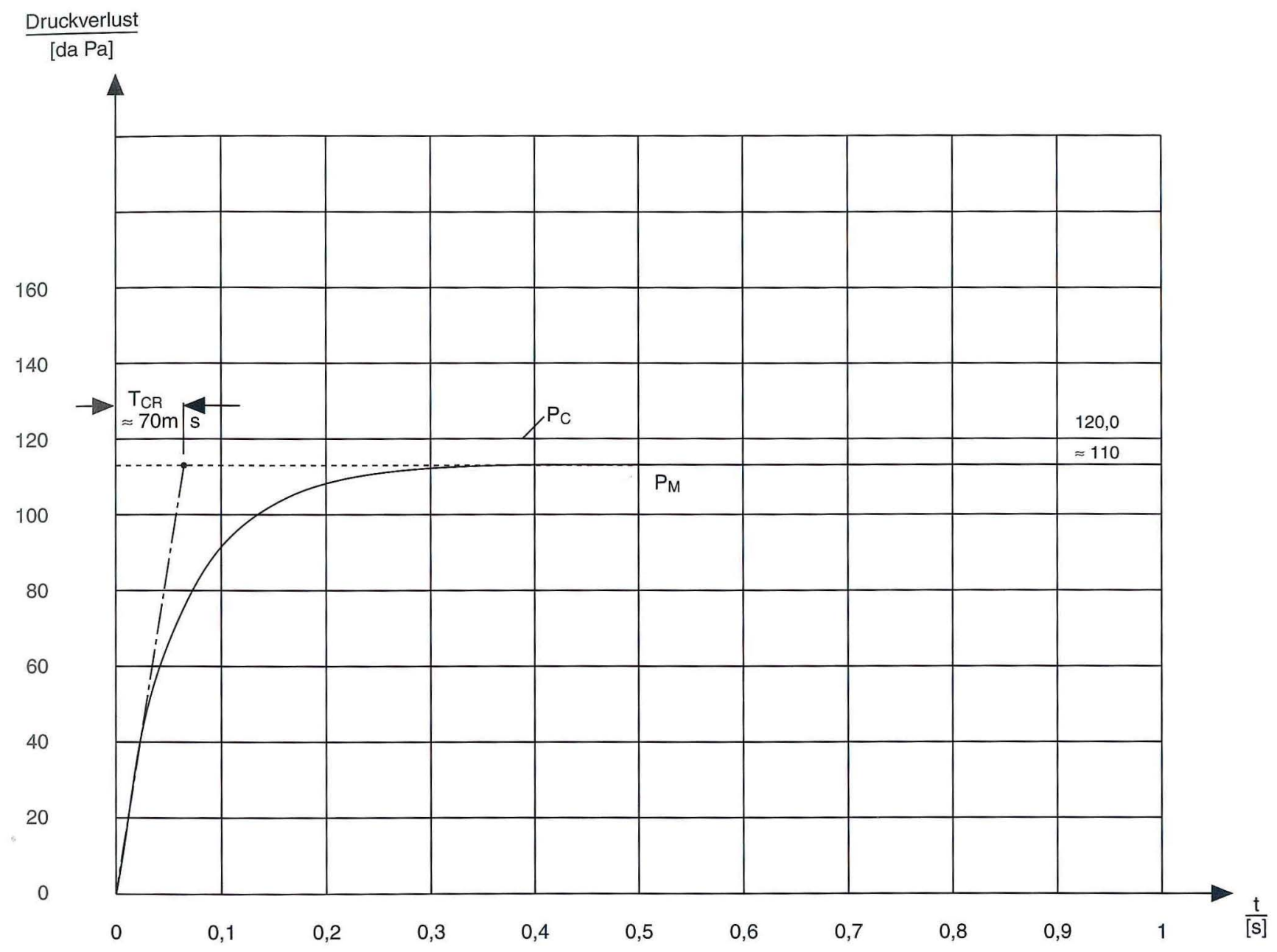

Abbildung 16.

Sprungfunktion als Hubvolumenprofil $\phi=17,5 \mathrm{ml} / \mathrm{s}$ für $t>0$. 


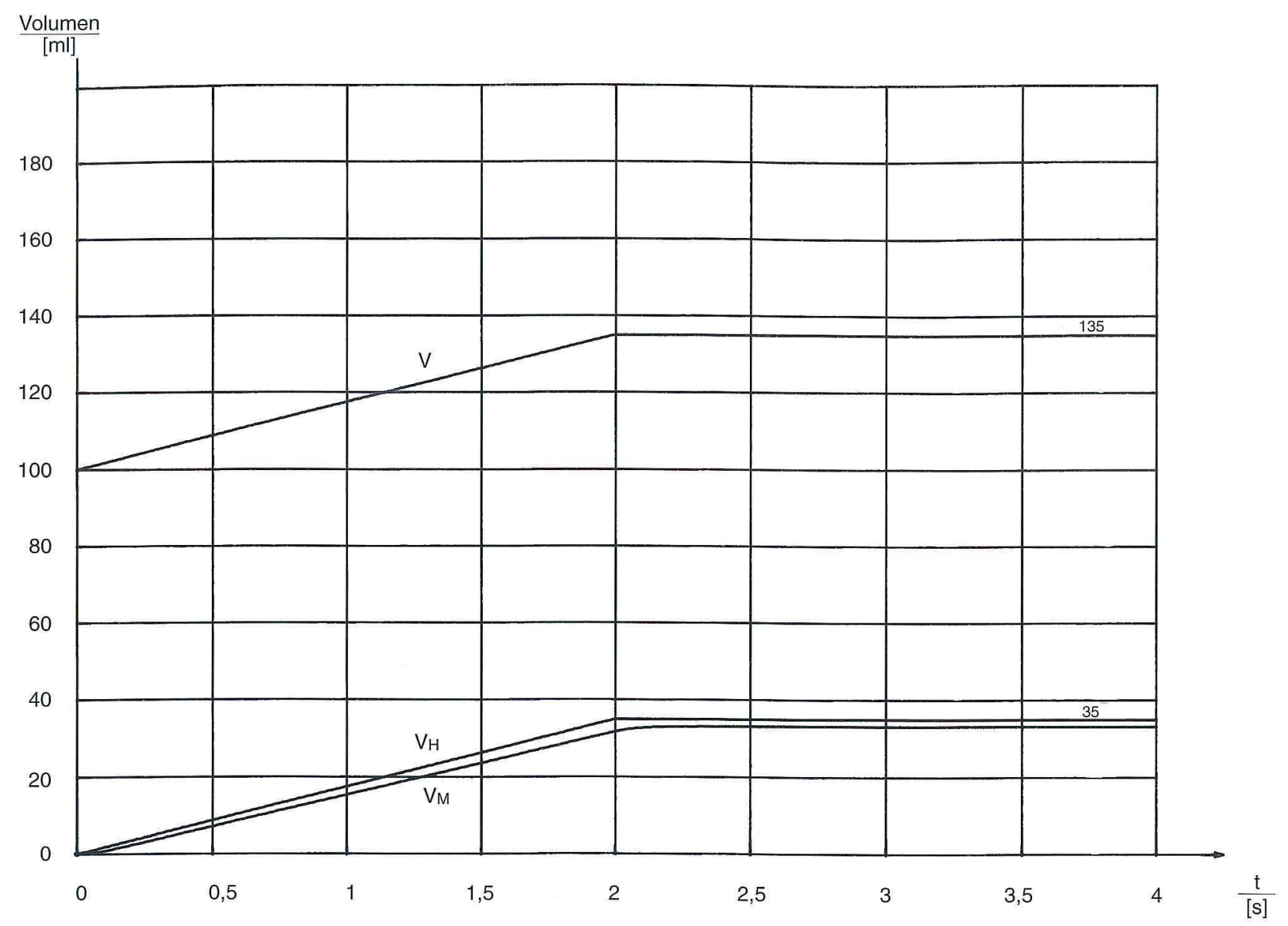

Volumenstrom

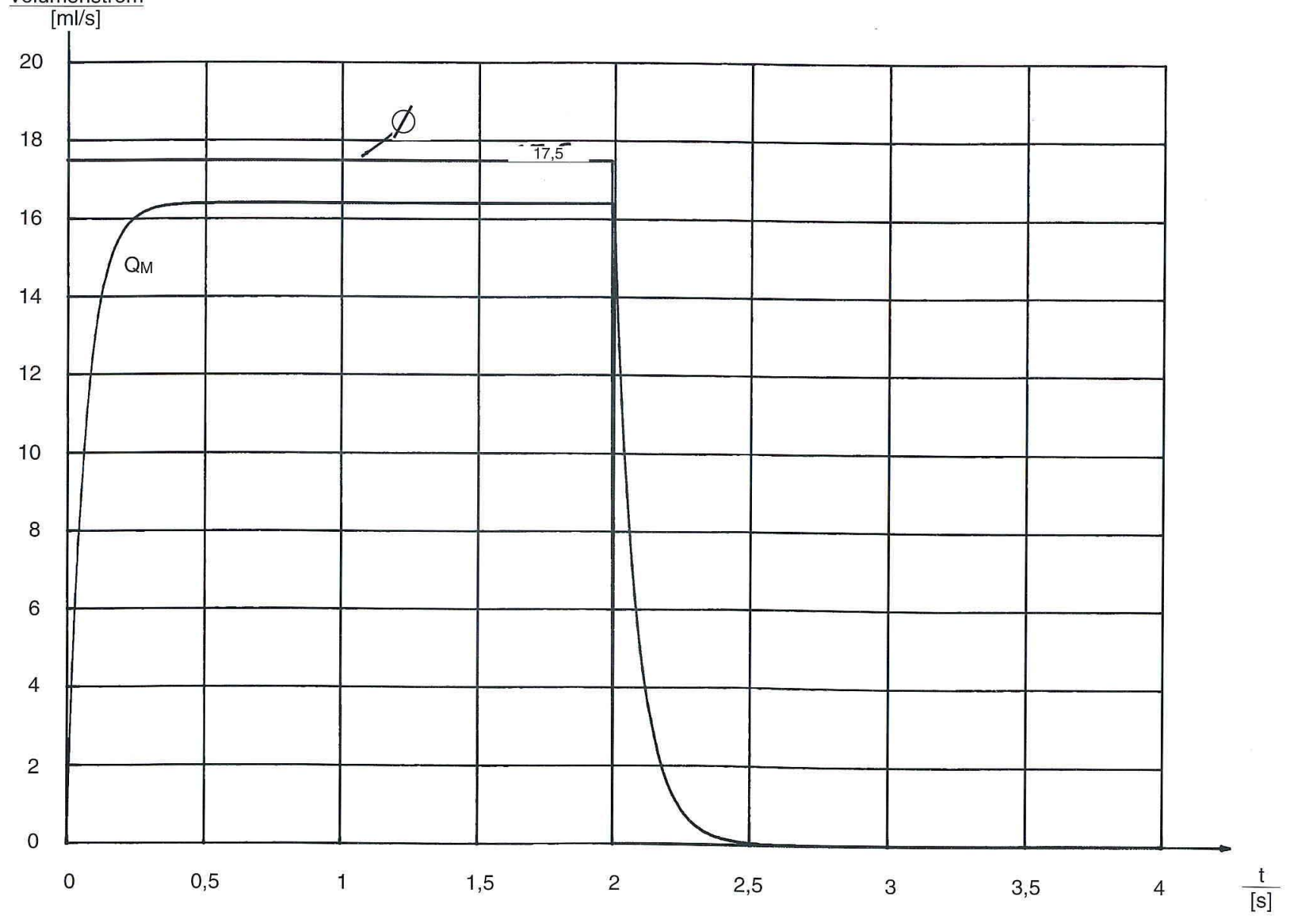




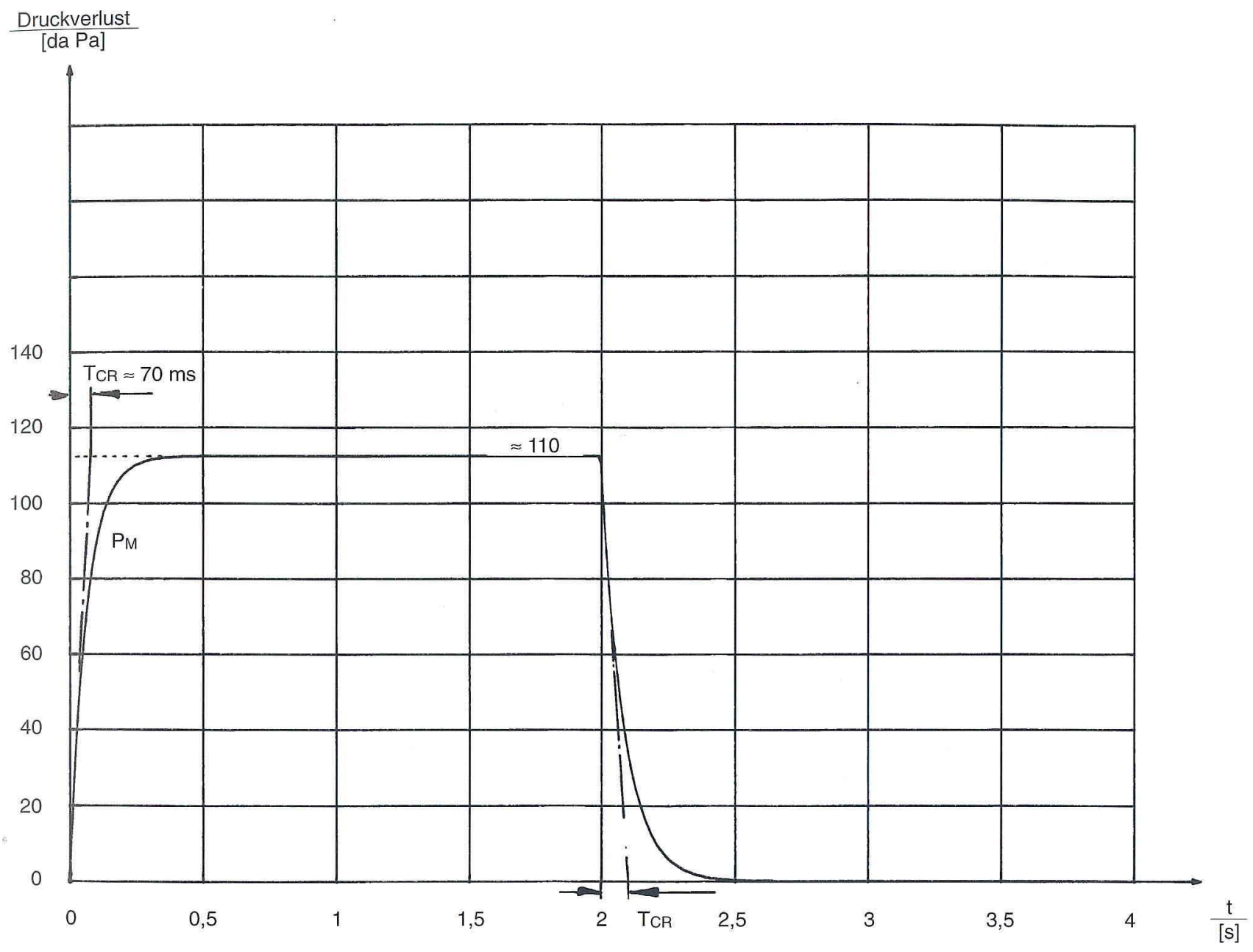

Abbildung 17.

Rechteckimpuls als Hubvolumenprofil: $\phi=17,5 \mathrm{ml} / \mathrm{s}$ für $0 \leq t \leq 2 \mathrm{~s}$ (gesamtes Hubvolumen $V_{H g}=35 \mathrm{ml}$ ). 

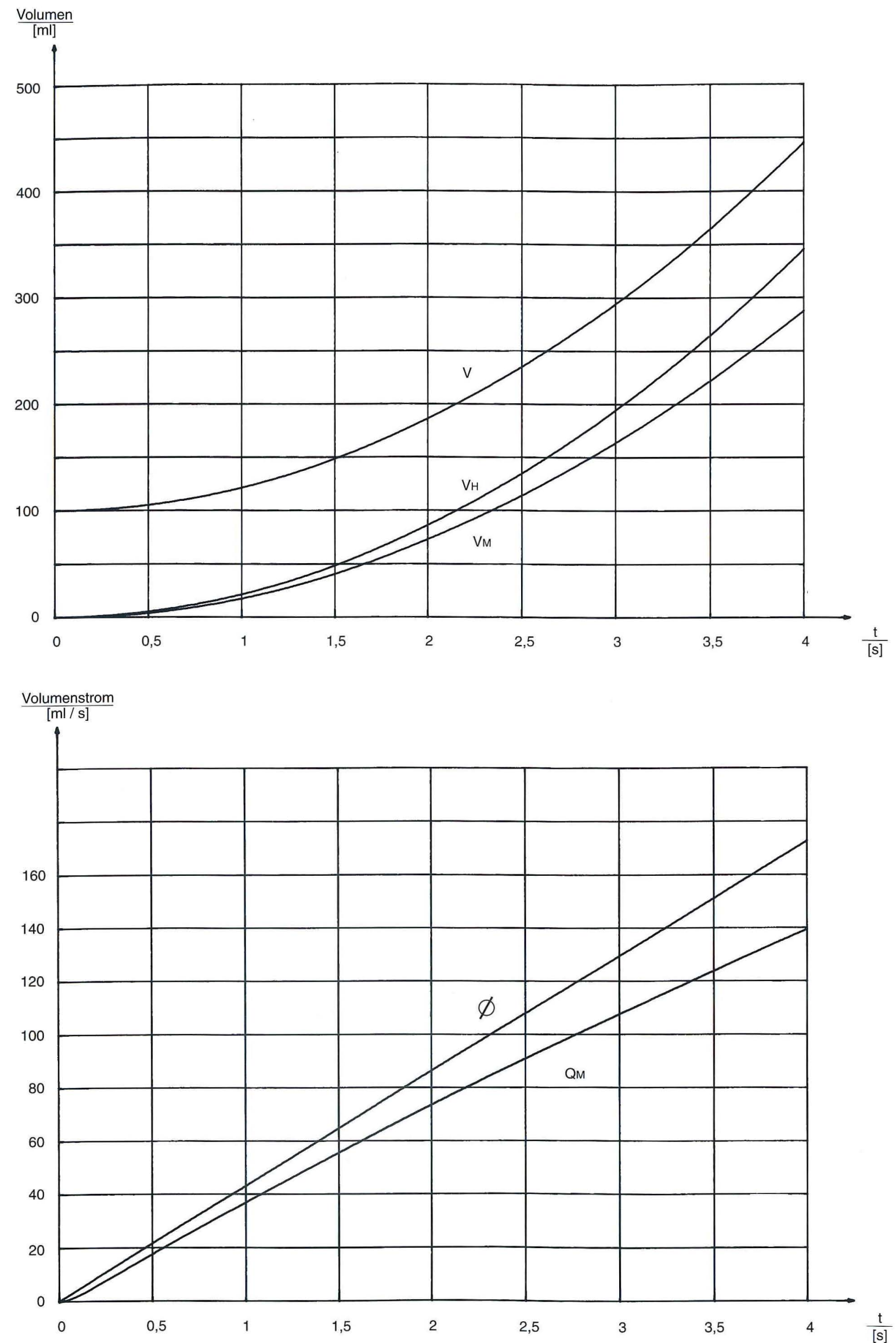


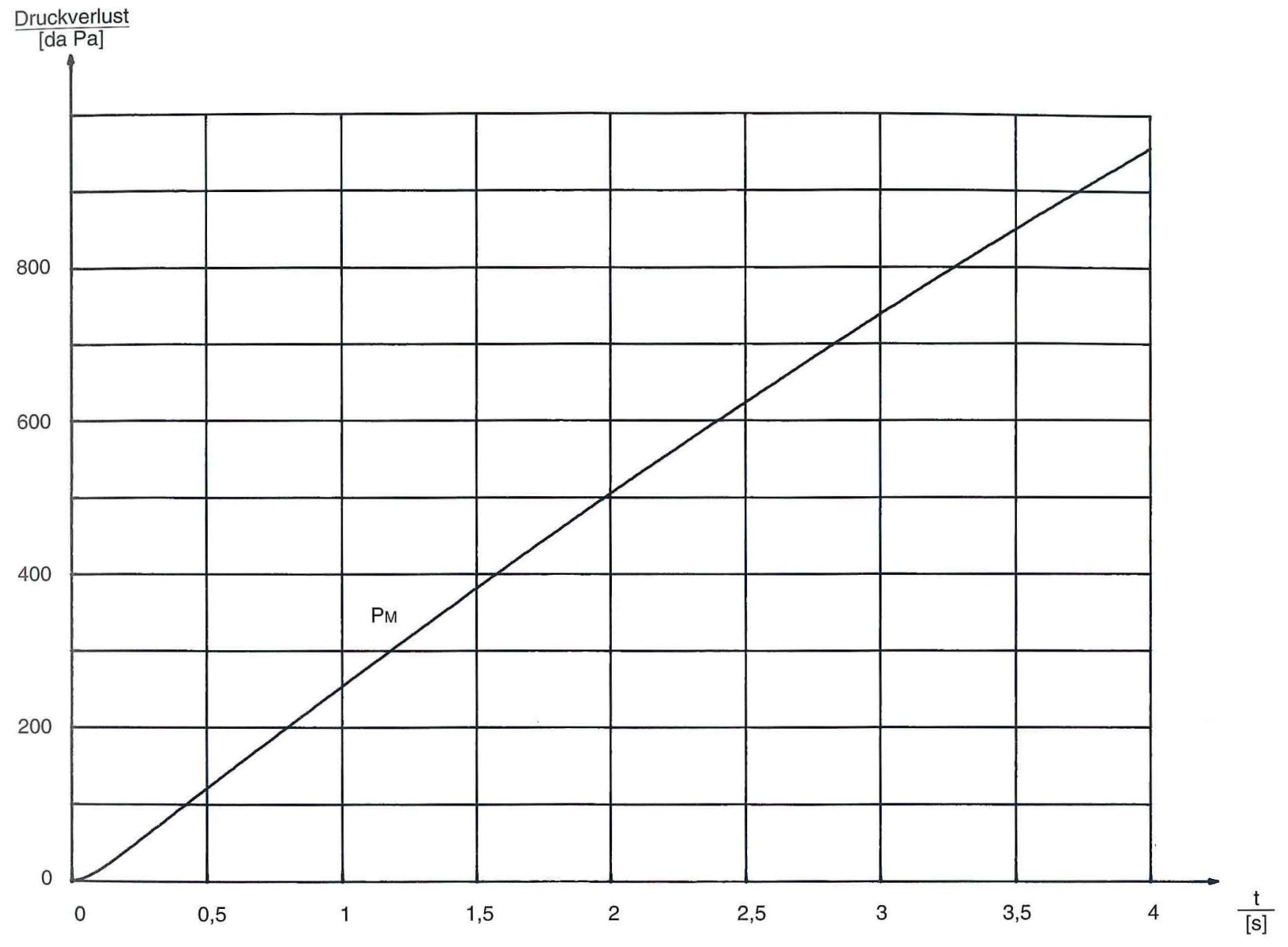

Abbildung 18.

Rampenfunktion als Hubvolumenprofil: $\phi=\alpha \cdot t$ für $t>0$ mit $\alpha=17,5 \cdot(\pi / 2)^{2} \mathrm{ml} / \mathrm{s}^{2}$ (Steigung bei glockenförmigem Hubvolumenprofil). 


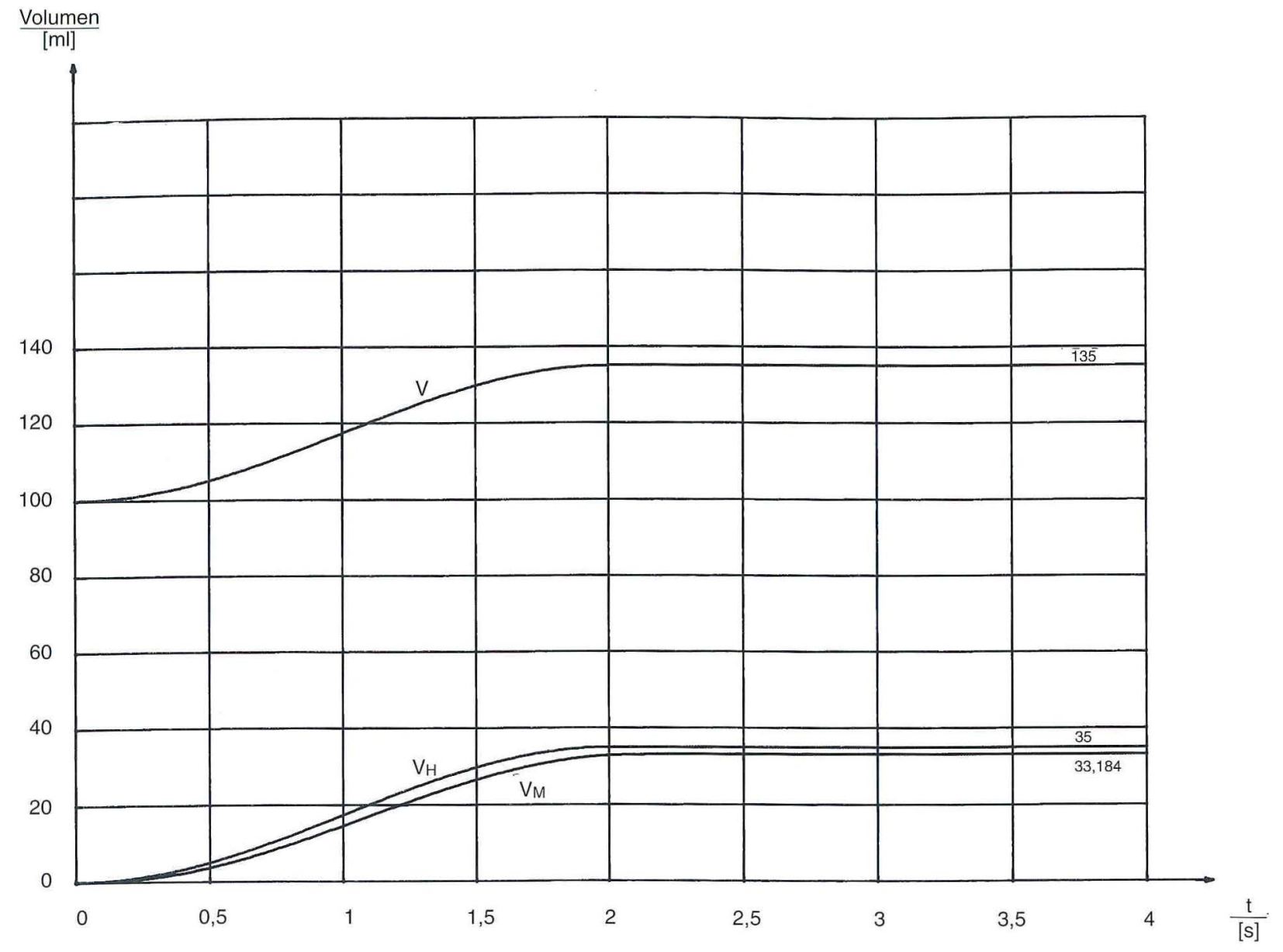

Volumenstrom

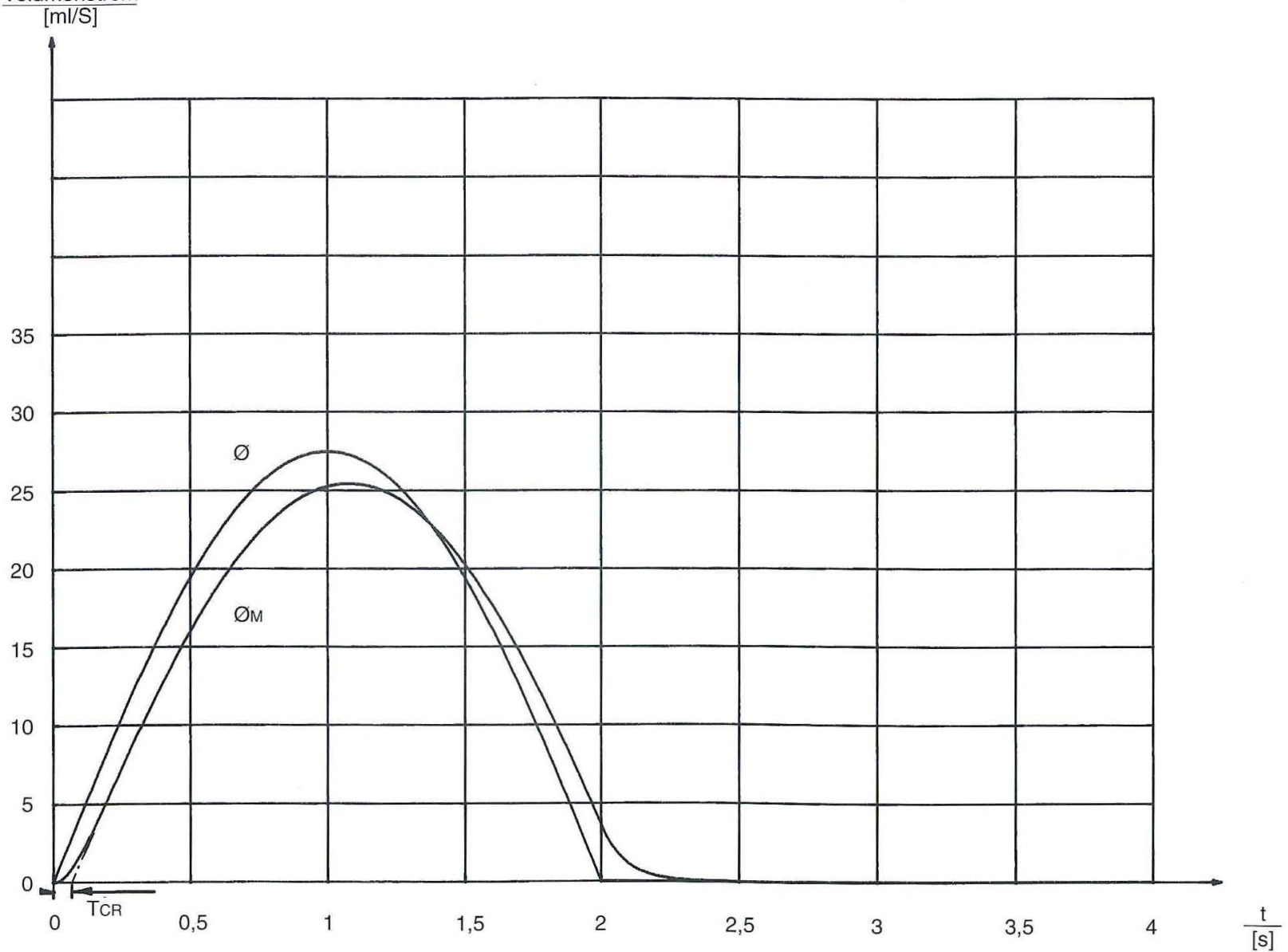




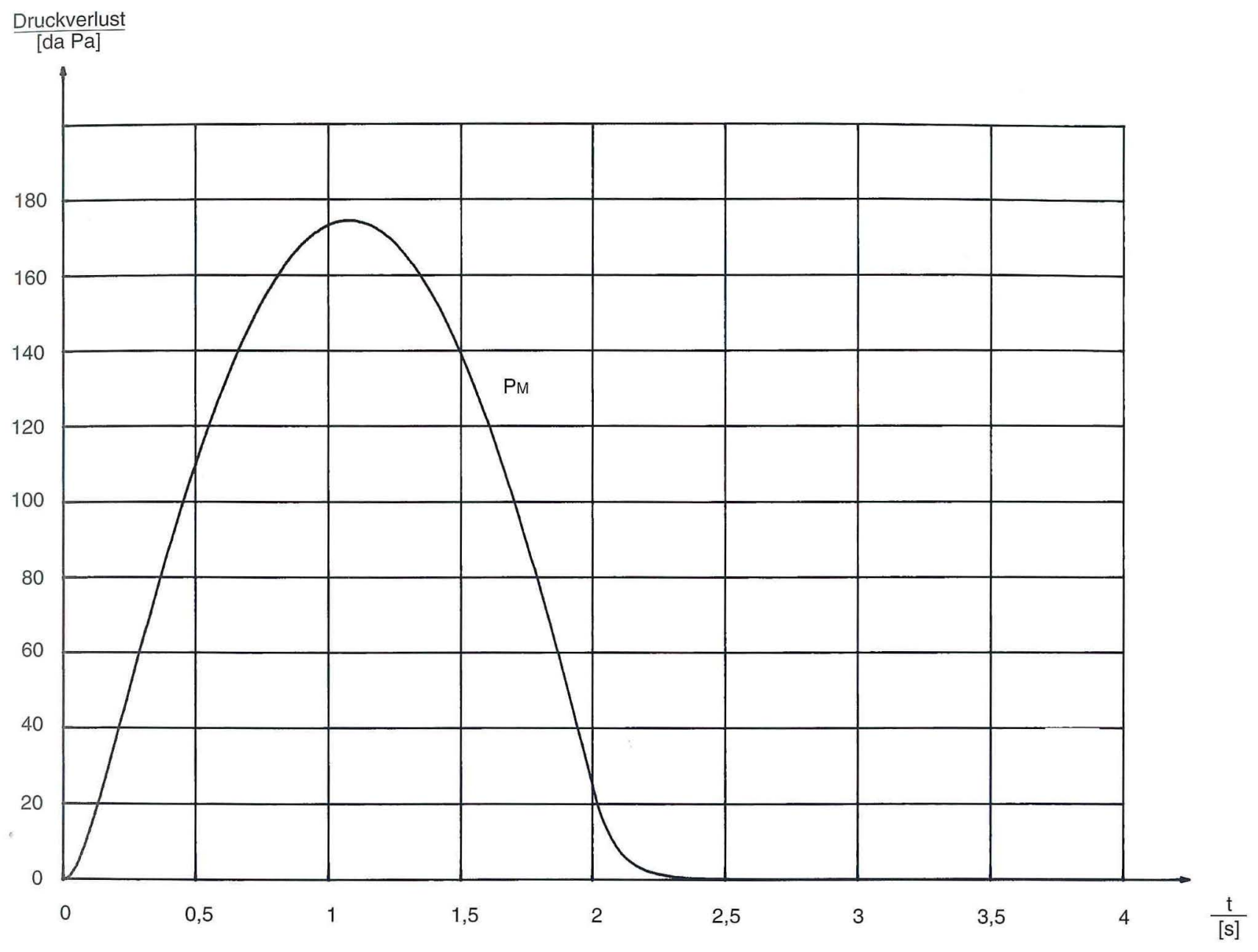

Abbildung 19.

Glockenförmiges Hubvolumenprofil $\phi(t)$ nach Gleichung [5.4] (gesamtes Hubvolumen $V_{H g}=35 \mathrm{ml}$ ). 

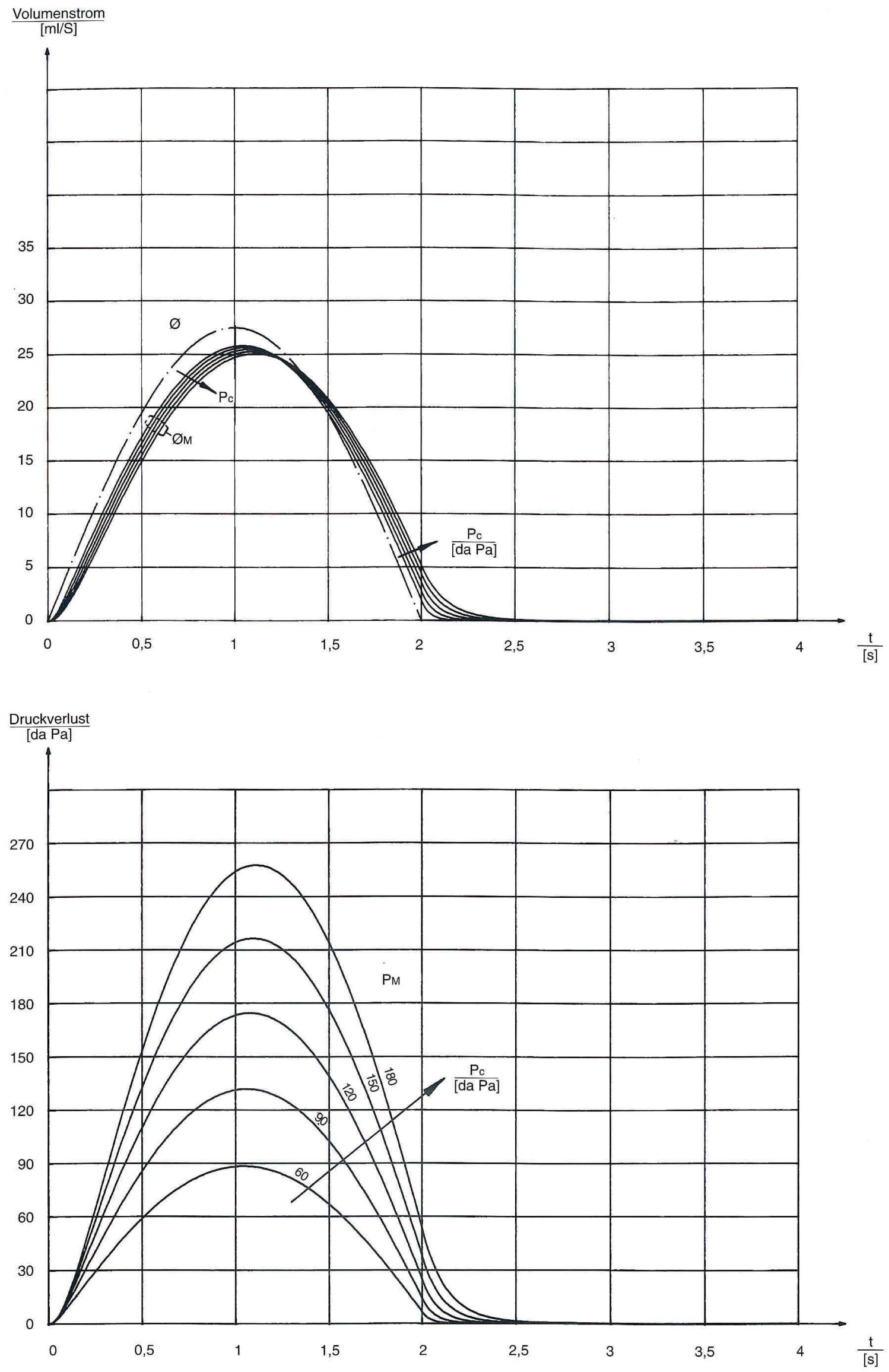

Abbildung 20.

Parametereinfluß des Druckverlustes der offenen Cigarette $P_{c}=60 / 90 / \underline{120} / 150 / 180 \mathrm{daPa}$. 


\subsection{Simulation und Prozeßanalyse}

Die Analyse des Prozeßmodells kann auf vielfältige Art und Weise geschehen, z.B. mit Hilfe eines Rechners (PC) und Software zur Simulation nichtlinearer dynamischer Systeme. Das Strukturbild (Signalflußdiagramm) ist die unmittelbare Aufbereitung des Prozeßmodells bei einem blockorientierten grafischen Konfigurieren, wie bei der verwendeten "XANALOG-Simulation and control design software" (Abbildung 15).

XANALOG (7) ist eine Arbeitsumgebung zum menügesteuerten Entwurf multivariabler Regelungssysteme, zur Modellbildung, zur Entwicklung von Parameterstudien und zur Signalverarbeitung.

In einer Analyse und Simulationsstudie werden verschiedene Testsignale für die Eingangsgröße "Hubvolumenprofil $\phi(t)^{\prime \prime}$ benutzt. Abbildung 16 zeigt eine Simulation mit einer Sprungfunktion, d.h.

$$
\phi(t)=\left\{\begin{array}{c}
\text { Null } \quad \begin{array}{r}
t \\
\text { für } \\
17,5 \mathrm{ml} / \mathrm{s} t
\end{array} \quad 0 .
\end{array}\right.
$$

Dies ist die Situation, wie sie bei der stationären Messung des Zugwiderstandes auftritt [3]. Als stationärer Endwert für den Druckverlust $P_{M}$ folgt jedoch nicht der Druckverlust der offenen Filter-Cigarette $P_{c}=120$ $\mathrm{daPa}$, sondern wegen der Systemstruktur bzw. des Übertragungsverhaltens

$$
\begin{gathered}
\lim _{t \rightarrow \infty} P_{M}(t)=\frac{1}{1+\frac{1}{\dot{C} \cdot Z_{M}}} \cdot p_{\mathrm{atm}} \\
=\frac{1}{1+\frac{\boldsymbol{R}_{G} \cdot \boldsymbol{v} \cdot \rho_{f} \cdot Q_{N Z}}{\phi \cdot P_{C}}} \cdot p_{a t m} \approx 110 \mathrm{daPa} .
\end{gathered}
$$

Die "Zeitkonstante" $T_{C R}$ läßt sich aus dem Diagramm für den Druckverlust $P_{M}$ (oder dem Zugprofil $Q_{M}$ ) als Anstiegszeit (rise time, step reponse time) ablesen:

$$
T_{C R} \approx 70 \mathrm{~ms} \text {. }
$$

Ist das Hubvolumenprofil ein Rechteckimpuls der Höhe $17,5 \mathrm{ml} / \mathrm{s}$ mit der Zeitdauer $t_{d}=2 \mathrm{~s}$, beträgt das gesamte Hubvolumen $V_{\mathrm{Hg}}=35 \mathrm{ml}$. Die Systemantwort ist in Abbildung 17 dargestellt und die "Zeitkonstante" $T_{C R}=70 \mathrm{~ms}$ läßt sich auch hier ablesen. Wählt man entsprechend Gleichung [5.5] als Testsignal eine Rampenfunktion $\phi=\alpha \cdot t$ mit $\alpha=17,5$. $(\pi / 2)^{2} \mathrm{ml} / \mathrm{s}^{2}$, erhält man Abbildung 18 .
Das Zugprofil $Q_{M}$ folgt im Zeitintervall $0 \leq t \leq 0,5 \mathrm{~s}$ dem Hubvolumenprofil $\phi$ asymptotisch im Abstand $\alpha$ - $T_{C R}$, die "Zeitkonstante" $T_{C R}$ ist auch hier ablesbar. Für $t>0,5 \mathrm{~s}$ wird der Einfluß des zeitvariablen Volumens durch die größerwerdende Abweichung des Zugprofils $Q_{M}$ bemerkbar.

Wird ein glockenförmiges Hubvolumenprofil wie in Gleichung [5.4] als System-Eingangsgröße verwendet, erhält man Abbildung 19.

In der Anfangsphase entspricht das Systemverhalten dem bei einer Rampenfunktion (Abbildung 18). Global ist eine Ähnlichkeit zur Rechteckfunktion (Abbildung 17) erkennbar und entspricht dem unter realen Bedingungen gemessenen Zugprofil bzw. Unterdruck (Druckabfall) $P_{M}$.

Einen erheblichen Einfluß auf die Dynamik hat die Ersatzströmungsimpedanz $Z_{M}$ nach Gleichung [5.7] bzw. der Druckverlust der offenen Cigarette (Zugwiderstand) $P_{M}=P_{c}$ nach Gleichung [5.8]. Eine Parametersimulation für $P_{c}=60 / 90 / \underline{120} / 150 / 180 \mathrm{daPa}$ $(1 \mathrm{daPa} \approx 1 \mathrm{mmWS}$ ) zeigt Abbildung 20.

In (1) wurde ausführlich der stationäre Strömungszustand in der Cigarette untersucht, auch mit dem Glutkegel $Z_{G}$ und der reduzierten Tabakstranglänge $l_{S r}$ infolge des Abrauchens. Folgende Ergebnisse sind dort unter anderem zu entnehmen (kanonisches Zahlenbeispiel):

1) Die Impedanz des Glutkegels im Verhältnis zum Wellenwiderstand $Z_{W}$ ist etwa $Z_{G} / Z_{W}=0,1$ bis 0,5 (Abrauchbedingungen).

2) Der Zugwiderstand (Druckverlust) der offenen Cigarette wurde mit $P_{M}=P_{c}=120 \mathrm{daPa}(120$ mmWS) angenommen. Im Moment des Anzündens springt der Druckverlust auf $P_{M}=130$ bis $160 \mathrm{daPa}$. Während des Abrauchens sinkt dieser Druckverlust monoton auf ca. $P_{M}=100$ bis 140 daPa (Abbildung 21). Die exakte Berechnung geschieht mit der Ersatz-Strömungsimpedanz des Tabakstranges $Z_{S r}$ (Gleichung [2.14], Abbildung 1d).

Eine dynamische Analyse der Fluidströmung der brennenden "warmen" Cigarette in der Abrauchmaschine ist mit Abbildung 20 sehr einfach möglich.

Bei der "kalten" Cigarette ist $P_{M}=P_{c}=120 \mathrm{daPa}$ zu wählen, im Augenblick des Anzündens ist $P_{M}=130$ bis $160 \mathrm{daPa}$ und damit die Umgebung des Parameters $P_{c}=150 \mathrm{daPa}$ zu beachten. Mit jedem Zug sinkt der Zugwiderstand, bis schließlich ca. $P_{M}=120 \mathrm{daPa}$ erreicht wird (Filter mit Stummel und Glutkegel). 


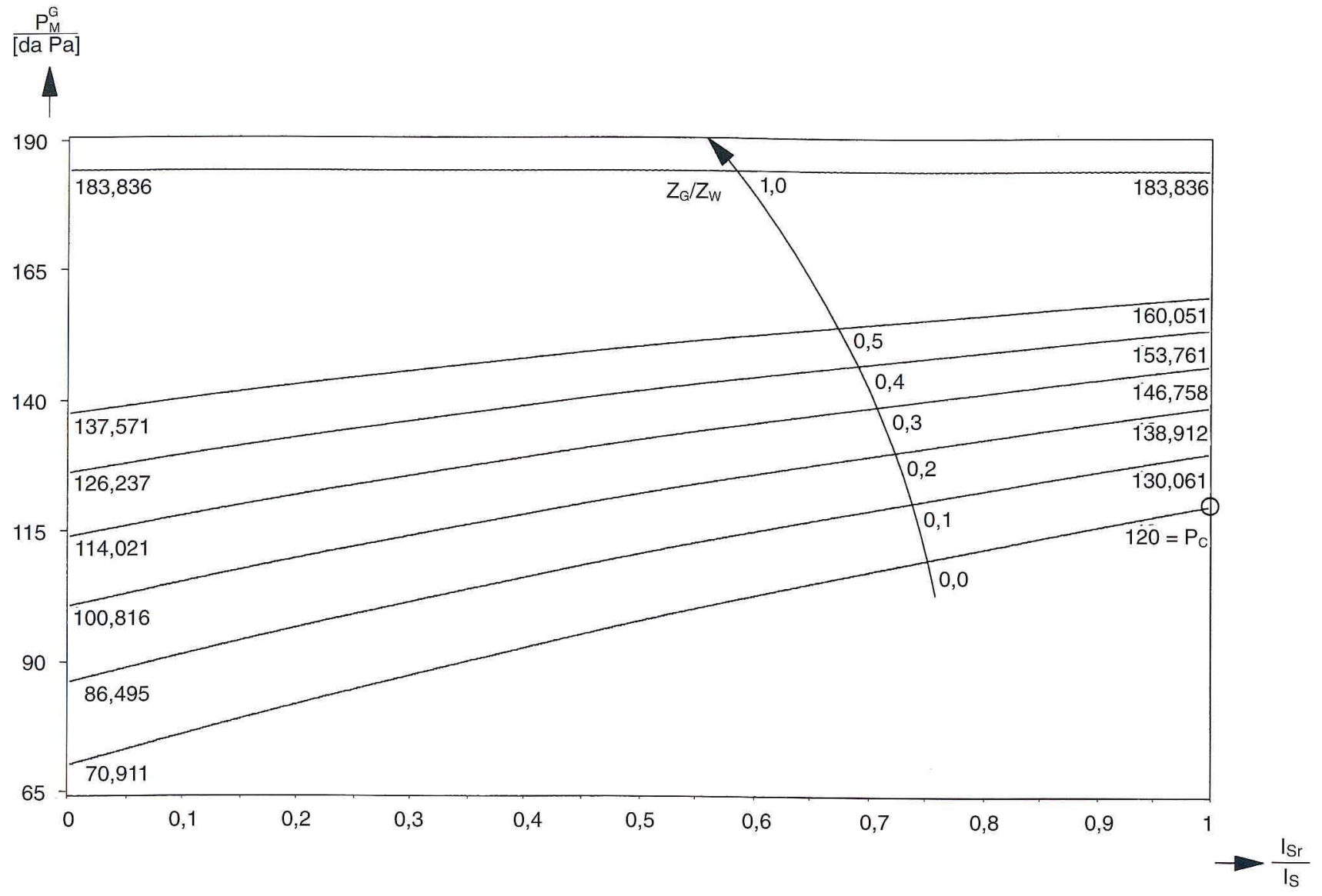

Abbildung 21.

Druckverlust der brennenden, unverschlossenen

Filter-Cigarette $P_{M}{ }^{G}$ in Abhängigkeit der reduzierten Tabakstranglänge $0 \leq I_{S} r<I_{S}$ und der Glutkegelimpedanz $Z_{G}\left(P_{M}{ }^{G}\right.$ $\left.=P_{M}\left(I_{S r}, Z_{G}\right)\right)$.

Die Paramtersimulation, wie in Abbildung 20 dargestellt, läßt sich daher für Betrachtungen von Cigaretten mit unterschiedlichem Zugwiderstand $P_{c}$ verwenden, aber auch für eine Berücksichtigung des Glutkegels und der reduzierten Tabakstranglänge bei jedem Zug.
Das Totvolumen $V_{T}$ muß so klein wie möglich sein; in den Simulationen wurde $V_{T}+\varepsilon \cdot V_{C i g}=100 \mathrm{ml}\left(V_{C i g}\right.$ $=4,17 \mathrm{ml}$ ) gewählt. Das Totvolumen $V_{T}$ ist ebenfalls signifikant für die Dynamik, gemäß Gleichung [5.6], [5.7] bzw. [5.8] mit $V=V_{H}+V_{T}+\varepsilon \cdot V_{C i g}$. Eine Parametersimulation für $V_{T}+\mathcal{E} \cdot V_{\text {Cig }}=25 / 50 / 75 /$ $\underline{100} / 125 / 150 \mathrm{ml}$ zeigt Abbildung 22 . 

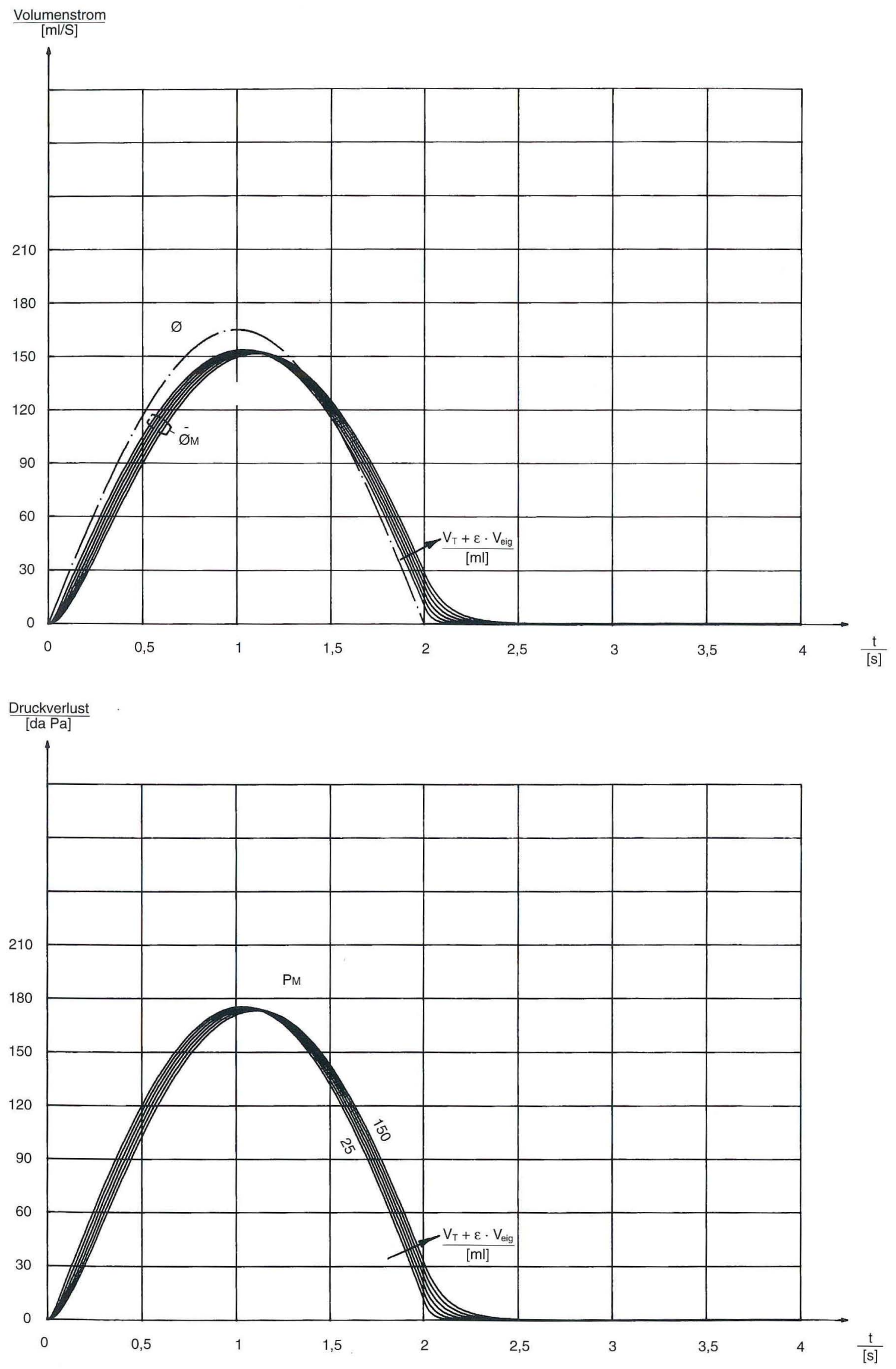

Abbildung 22.

Parametereinfluß des Totvolumens $V_{T}$ der Rauchmaschine inklusive Cigarettenfluidvolumen $\varepsilon \cdot V_{\text {cig }}$. $V_{T}+\varepsilon \cdot V_{\text {cig }}=25 / 50 / 75 / \underline{100 / 125 / 150 \mathrm{ml}}$ 


\section{REFERENCES}

1. Gaißer, H.: Die stationäre Strömung in der Cigarette und deren Einfluß auf den Zugwiderstand (mit ausführlichem Literaturverzeichnis, insbesondere zur Theorie dynamischer Systeme); Beitr. Tabakforsch. Int. 15 (1992) 93-122.

2. DIN/ISO 3308, Zigaretten-Abrauchmaschine für Routineanalysen, Begriffe und Standardbedingungen; Beuth, Berlin, 1992.

3. DIN 10251, Bestimmungen des Zugwiderstandes, Begriffe und Bedingungen; Beuth, Berlin, 1986.

4. Hegges, P.J.: Fixed beds - fluid mechanics and heat tranfer (Head exchanger design handbook, Bd. 2; VDI, Düsseldorf, Hemisphere, New York, 1986.

5. Brauer, H., and D. Mewes: Stoffaustausch, ein schließlich chemischer Reaktion; Sauerländer, Frankfurt a.M., 1971.

6. Grassman, P.: Physikalische Grundlagen der Verfahrenstechnik; Sauerländer, Frankfurt a.M., 1983.

7. XANALOG, nonlinear dynamic systems: modeling, simulation and realtime controls testing-software; XANALOG, Woburn, 1991
Danksagung

Die Anfertigung des Manuskriptes auf dem PC, Text mit Word, Abbildungen mit Freelance und Simulationsdiagramme mit XANALOG haben Studenten im Fachpraktikum mit Gescbick und Scbönheitsgefïhl übernommen. Dafür, wie auch für Ibre Mitarbeit beim Korrekturlesen, sei den Herren $R$. Bruns und A. Trinkies an dieser Stelle aufrichtig gedankt.

Anscbrift des Autors

Horst Gaißer

BAT Cigarettenfabriken GmbH

Technische Planung

Babrenfelder Chaussee 139

22761 Hamburg 